Review

\title{
Exposure to per- and polyfluoroalkyl substances and premature skin aging
}

\author{
Sayed Esmaeil Mousavi ${ }^{\text {a, *, Juana Maria Delgado-Saborit }}$ b,c,d,e, Lode Godderis ${ }^{\text {f,g }}$ \\ ${ }^{a}$ Department of Water and Wastewater Treatment, Water and Wastewater Consulting Engineers (Design \& Research), Isfahan, Iran \\ ${ }^{\mathrm{b}}$ Universitat Jaume I, Perinatal Epidemiology, Environmental Health and Clinical Research, School of Medicine, Castellon, Spain \\ ${ }^{\mathrm{c}}$ ISGlobal Barcelona Institute for Global Health, Barcelona Biomedical Research Park, Barcelona, Spain \\ ${ }^{\mathrm{d}}$ Environmental Research Group, MRC Centre for Environment and Health, Imperial College London, United Kingdom \\ ${ }^{\mathrm{e}}$ Division of Environmental Health \& Risk Management, School of Geography, Earth \& Environmental Sciences, University of Birmingham, Birmingham, United Kingdom \\ ${ }^{\mathrm{f}}$ Laboratory for Occupational and Environmental Hygiene, Unit of Environment and Health, Department of Public Health and Primary Care, KU Leuven, 3000 Leuven, \\ Belgium \\ ${ }^{\mathrm{g}}$ IDEWE, External Service for Prevention and Protection at Work, Interleuvenlaan 58, 3001 Heverlee, Belgium
}

\section{A R T I C L E I N F O}

\section{Editor: Dr. R. Teresa}

\section{Keywords:}

Dermal absorption

PFASs bioaccumulation

Oxidative stress

DNA damage

Telomere shortening

Skin integrity

\begin{abstract}
A B S T R A C T
Per- and polyfluoroalkyl substances (PFASs) are a ubiquitous group of persistent chemicals distributed globally in the environment. Skin aging is a notorious process that is prematurely induced by the interaction between endogenous and exogenous factors, including exposure to environmental chemicals. The existing evidence suggests that skin absorption of PFASs through dermal contact may be an important route of exposure to these chemicals in humans. On the other hand, PFASs intake by other routes may lead to PFASs bioaccumulation in the skin via tissue bio-distribution. Additionally, the presence of PFASs in consumer and cosmetic products combined with their daily close contact with the skin could render humans readily susceptible to dermal absorption. Therefore, chronic low-dose dermal exposure to PFASs can occur in the human population, representing another important route of exposure to these chemicals. Studies indicate that PFASs can threaten skin health and contribute to premature skin aging. Initiation of inflammatory-oxidative cascades, induction of DNA damage such as telomere shortening, dysregulation of genes engaged in dermal barrier integrity and its functions, signaling of the mitogen activated protein kinase (MAPK) pathway, and last but not least the down-regulation of extracellular matrix (ECM) components are among the most likely mechanisms by which PFASs can contribute to premature skin aging.
\end{abstract}

\section{Introduction}

Since the 1940s, per- and polyfluoroalkyl substances (PFASs), a large group of persistent organic pollutants (POPs), have been extensively dispersing in the environment worldwide (Nakayama et al., 2019). These chemicals are categorized into two main subgroups, including perfluoroalkyls and polyfluoroalkyls. The former encompasses an enormous family of the fluorinated compounds within which the carbon chains are fully occupied by fluorine atoms. These chains commonly terminate with a carboxylate (e.g. perfluorooctanoic acid (PFOA), perfluordecane sulfonic acid (PFDS)), or sulfonate group (e.g. perfluorooctane sulfonic acid (PFOS), perfluorohexane sulfonic acid $\left.\left(\mathrm{PFH}_{\mathrm{X}} \mathrm{S}\right)\right)$. On the contrary, in polyfluoroalkyls, the carbon chains are not fully fluorinated. Plenty of fluoropolymers and fluorotelomers fall into this subset. They are eventually transformed or metabolized to perfluoroalkyl acids and carboxylates (Li et al., 2019).

Thanks to the perfluoroalkyl moiety $\left(\mathrm{C}_{n} \mathrm{~F}_{2 n+1^{-}}\right)$, these highly bioaccumulative compounds benefit from prominent physico-chemical

Abbreviations: AD, atopic dermatitis; APFO, ammonium perfluorooctanoate; arNOX, age-related NADH oxidase; CE, cornified envelope; DSBs, double-strand

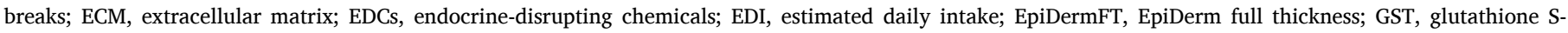

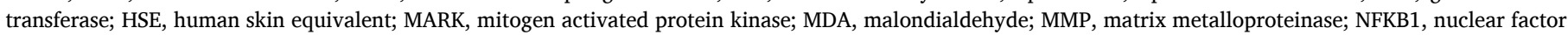

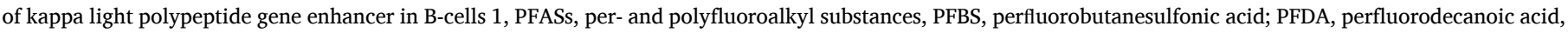

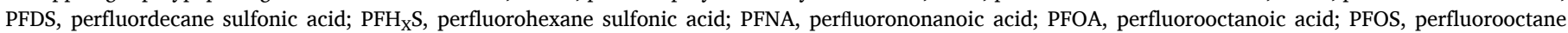

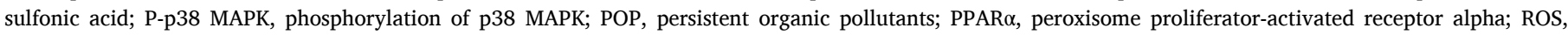
reactive oxygen species; SA- $\beta$-gal, senescence-associated $\beta$-galactosidase; SC, stratum corneum; VDES, vitamin D endocrine system.

* Corresponding author.

E-mail address: esmaeil.s.mousavi@gmail.com (S.E. Mousavi). 
features such as stronger acidity, higher surface activity, chemical and thermal stability, and water-and oil-repellency compared with their hydrocarbon counterparts (Wang et al., 2017). Such unique properties make them unusually exceptional (if no incomparable) to be utilized in a very wide spectrum of industries and products. There is a myriad of these fluoroalkyl compounds all of which have considerable similarities in structure, behavior, and effects (Barzen-Hanson et al., 2017). Extensive application and resistance to natural decomposition have dispersed PFASs throughout the globe. As can be seen in Fig. 1, all the steps from production, consumption, to waste treatment and disposal of PFASs have the potential to release these chemicals or their precursors into the environmental compartments, including the atmosphere, hydrosphere, and lithosphere. Environmental distribution of PFASs in turn, leads to their exposure to living organisms and their bioaccumulation through the trophic chains. This is an indirect route of exposure affecting humans and other forms of life. On the other hand, exposure to consumer products containing PFASs is considered a direct route of exposure increasing the risk of health effects.

Due to the ubiquity of PFASs and the serious threats that they continuously pose to the environment and people, the attention to the environmental fate and transport, as well as the toxicity of these chemicals has been continuously increasing over the last few decades. Based on findings obtained from growing epidemiological and toxicological studies on PFASs, exposure to these long-lasting chemicals participates in a wide range of adverse health outcomes, including endocrine disruption, immunotoxicity, developmental disorders, and some types of cancers (ATSDR, 2018; Temkin et al., 2020). That is why an issue of high importance turning into a priority is lowering the use and exposure to long-chain PFASs through the innovation and development of fluorine-free alternatives. It is worth noting a recent growing concern is related to exposure to some short-chain PFASs which have been considered as a replacements for long-chain PFASs (Wang et al., 2013, 2015).

Encompassing the tremendous growth of industrialization, an increasing number of pollution sources release hazardous chemicals into the environment. For this reason, measuring exposure to environmental pollutants, under the umbrella term of "exposome", has recently turned into an ineluctable constituent of epidemiological analysis assessing the linkage between environmental exposure and individual health and pathogenesis of a large number of human diseases. However, the influence of environmental chemicals on skin health is less studied compared with other health endpoints. During the last two decades, the

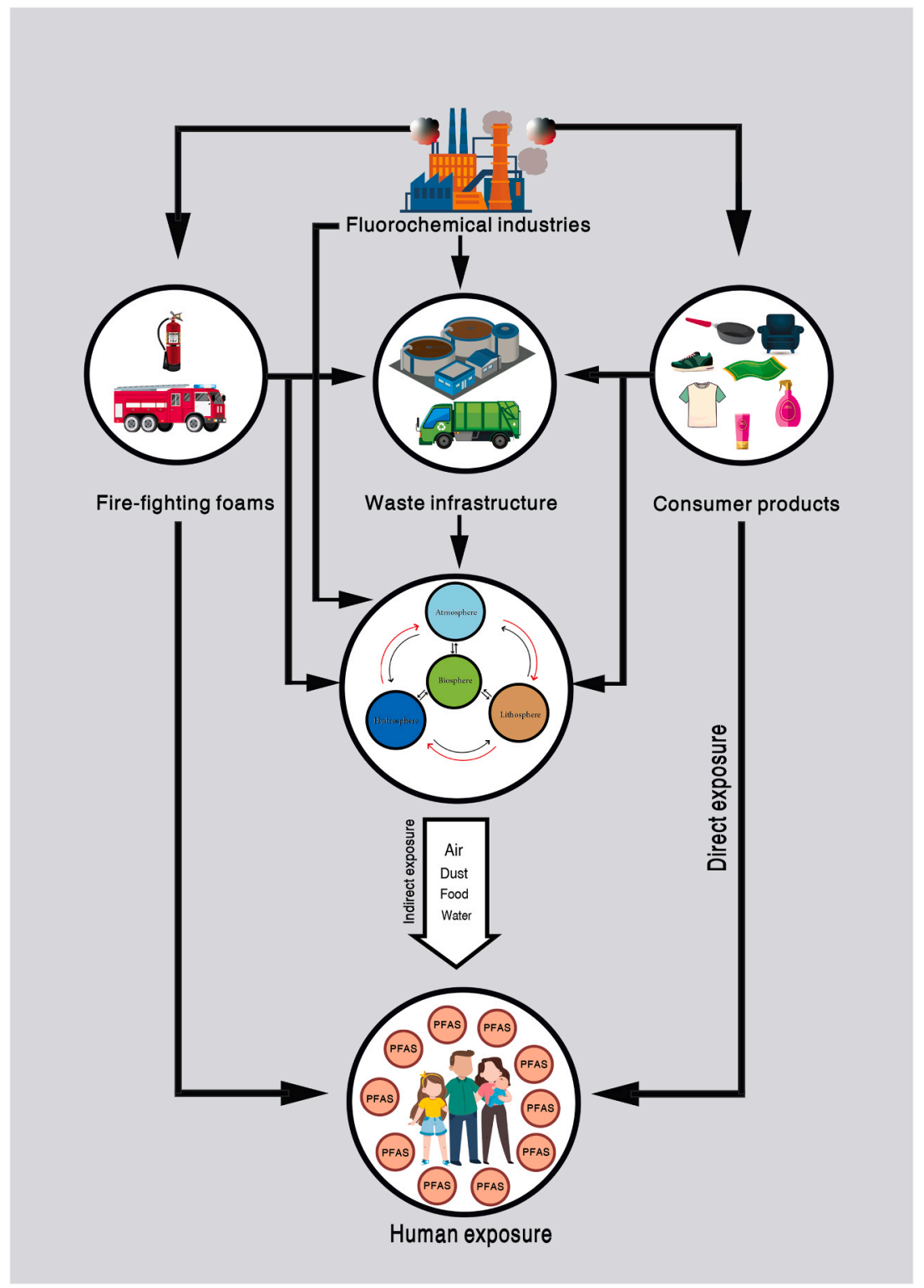

Fig. 1. Presence and dispersion of PFASs in the environment. After the production of PFASs by fluorochemical manufacturers, they distribute across the environment via the integrated cycles in which atmosphere, hydrosphere, and lithosphere are interconnected. Besides, the leachate and gaseous emissions from landfills, the effluent of wastewater treatment plants, and the release of fire-fighting foams render the environment much more susceptible to PFASs. Air, water, and soil interact with each other in an interconnected way and transfer PFASs to the biosphere, including macro- and microorganisms. As a result, these non-biodegradable compounds penetrate the biosphere and accumulate across the trophic chains. Such a chain of events results in indirect exposure of humans to PFASs via air, dust, food, and drinking water. Humans are additionally exposed to consumer products containing PFASs and their precursors. The other, but considerably less frequent, direct route of exposure is by dermal contact and inhalation of fire-fighting foams. 
detrimental effects of environmental contaminations on the human skin emerged as a new domain of environmental medicine. Specifically, a special emphasis has been placed on the contribution of air pollutioninduced skin diseases and skin aging. Furthermore, toxic impacts of dermal exposure to POPs, especially in the workplace, have been studied substantially. Concerning PFASs exposure, although numerous experimental and epidemiological studies have been conducted for understanding the possible contributions of these chemicals to the pathogenesis of a broad array of diseases, the interaction between PFASs and the skin has received less attention as their dermal toxic effects may have been underestimated.

Of all the environmentally-induced skin disorders, skin aging is a prevalent global health challenge requiring serious attention. In this regard, the term "extrinsic skin aging" has been proposed to cover the cutaneous aging stemmed from environmental stressors. It is worth mentioning that the dermal absorption of pollutants is capable of triggering skin damage and bringing about undesirable structural changes. Within the last decade, some experimental studies have been designed to consider dermal exposure to PFASs. For the first time, this review aims to critically and mechanistically examine existing evidence related to dermal effects induced by exposure to PFASs and to figure out potential pathways that may accelerate the process of skin aging.

\section{Methodology}

Briefly, the authors performed a literature review over PubMed Database on April 10th, 2020 using a combination of the following keywords: ("Fluorinated hydrocarbons", per- and polyfluoroalkyl substances", "PFASs", "perfluorooctanoic acid", "perfluorooctane sulfonic acid") AND ("skin"). Rayyan, a review web application, has been employed for making decisions in compliance with following exclusion and inclusion criteria (Ouzzani et al., 2016).

Original human and animal studies were included if meeting one of the following requirements: (1) reporting absorption, distribution, and accumulation of PFASs in the skin; (2) evaluating the toxic effects of PFASs under dermal exposure.

Studies were excluded if (1) Toxic endpoints of PFASs alternatives were investigated. (2) Toxic impacts of fluorinated hydrocarbons other than PFASs were examined. (3) Referred to non- dermal exposure. (4) Were written in languages other than English. (5) Referred to in-silico investigations.

To compile all available relevant studies, Google Scholar was used as an additional source to check the first 200 results for all possible combinations of the foregoing keywords. Although only original articles were included, the reference lists of relevant review articles were checked thoroughly to prevent missing any relevant items. Gray literature and research from governmental organizations and authorities published in journals other than academic ones, were excluded. The search strategy is presented in online Supplementary File 1.

\section{Results and discussion}

The following sections present findings extracted from the retained articles and are presented in a logical and interconnected order. Firstly, dermal exposure pathways to PFASs are considered, then their tissue distribution and bioconcentration are discussed with an emphasis on the skin. Finally, experimental and epidemiological evidence are examined to elucidate the most outstanding mechanisms triggering the procedure of skin aging under dermal exposure to PFASs.

\subsection{Dermal exposure pathways to PFASs}

Fig. 2 depicts the most significant exposure pathways to PFASs. In the case of dermal uptake of PFASs, direct contact of the skin surface with consumer products is the main route. PFASs exist in a myriad of consumer products that have direct contact with the human skin. Thanks to their versatility, PFASs are widely used to render shoes, clothes, carpets, sofa, to name but a few, water, soil, and stain-resistance. They

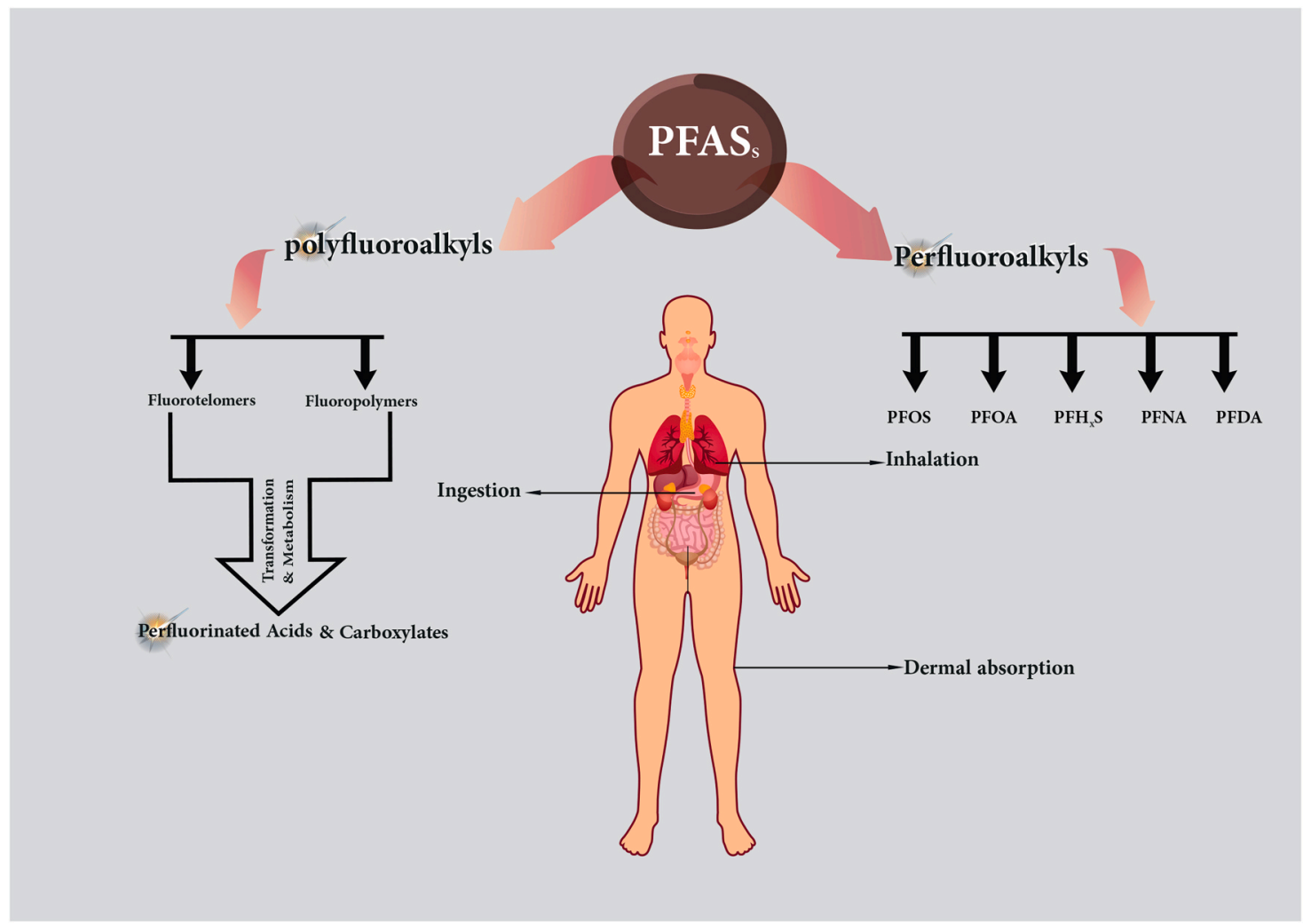

Fig. 2. Human exposure to PFASs. Human exposure pathways to PFASs mainly consists of air and dust inhalation, food and water ingestion, and dermal absorption. 
are utilized to prevent food sticking onto cookware and food packaging. Using long-chain PFASs -and recently their short-chain alternatives- in varnishes, lubricants, detergent products, textiles, paper, ink, waxes, and fire-fighting foams make the human health in general and the skin in particular frequently susceptible and vulnerable to these chemicals (Figs. 1 and 2).

PFASs are low molecular weight surfactants that easily penetrate the skin. Such a key feature is exploited in manufacturing cosmetic products as well. According to a comprehensive risk assessment carried out by the Danish Environmental Protection Agency, PFASs are found at high concentrations in roughly one-third of cosmetic and personal care products (Danish, 2018). It has been reported that exposure to PFOA and PFOS can interfere with membrane and barrier permeability in mitochondria (Hagenaars et al., 2013), brain (Chang et al., 2009), and skin (Fasano et al., 2005). Increasing dermal permeability may develop to dermal penetration and consequently dermal absorption of the chemicals. There are a very limited number of studies that have estimated the human exposure to PFASs through skin contact. Su and colleagues measured several perfluoroalkyl acids (PFAAs) in outdoor and indoor dust around a mega fluorochemical industrial park and assessed their estimated daily intake (EDI) for the local residents (Su et al., 2016). Dermal exposure to PFASs is estimated according to Eq. (1) (Poothong et al., 2019, 2020).

$\mathrm{EDI}_{\text {dermal }}=\frac{\mathrm{Q}_{\mathrm{hw} \times \mathrm{t}_{\mathrm{exp}} \times \mathrm{F}_{\text {uptake-dermal }}}}{\mathrm{BW}}$

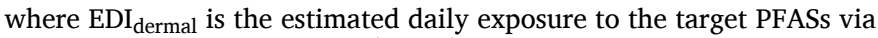
dermal absorption ( $\mathrm{pg} \cdot \mathrm{kg} \mathrm{bw}^{-1} \cdot \mathrm{day}^{-1}$ ), $\mathrm{Q}_{\mathrm{hw}}$ is the total mass present of the target PFASs (pg), $t_{\exp }$ is the exposure duration (day), $\mathrm{F}_{\text {uptake-dermal }}$ is the uptake fraction of PFASs absorbed via the skin, and BW is the bodyweight of an exposed individual (kg). Su et al. (2016) study reported that the EDI (ng/kg.bw/day) of PFAAs via dermal absorption and ingestion of dust contaminated with PFAAs was dependent on the age group and the distance between the population and the industrial park. Additionally, EDIs through the dust ingestion was evaluated to be roughly 4-14 times larger than that through skin contact (Su et al., 2016). Similarly, other studies indicated the dermal route, restricted to the hand's skin, was the least significant exposure to PFASs (Poothong et al., 2019, 2020). Nonetheless, dermal exposure to PFASs could be more substantial than what has been reported provided the whole surface of an individual's skin is considered. Moreover, attention should be paid to the fact that the skin is the only tissue that can be exposed to PFASs both externally and internally. On the one hand, PFASs can enter the body through ingestion and inhalation and transfer to the skin by the circulatory system and accumulate there during tissue distribution. On the other hand, skin can be exposed directly to PFASs through dermal contact. Therefore, despite initial estimations implying that exposure to PFASs through the skin is not comparable to other routes (dietary, inhalation, and dust ingestion), this issue is not incontrovertible. Developing more reliable and holistic methods are recommended to offer an actual and precise assessment of dermal exposure to PFASs.

\subsection{PFASs exposure and tissue distribution}

PFASs, by nature, are markedly stable and resistant to chemical reactions. In spite of being metabolically inert, these compounds can exert negative influences on endogenous metabolic processes. Disturbance in metabolism may induce biochemical alterations that are interconnected to systemic toxicity (DeWitt, 2015). Since PFASs are not metabolized in humans and animals, they are excreted with different half-lives through different routes. The half-life of PFASs in human serum has been estimated to be $8.3 \mathrm{yr}, 5.4 \mathrm{yr}$, and $3.8 \mathrm{yr}$ for PFHxS, PFOS, and PFOA, respectively (Olsen et al., 2008).

Tissue distribution refers to a process in which a chemical, primarily a drug, transfers from one site to another within the body. During this process, different doses of a chemical are delivered to each target organ by the vascular system. The residence time, distribution level, and elimination rate of a chemical is a complicated equation influenced by several variables, including but not limited to species, sex, and age (DeWitt, 2015). Assessment of tissue distribution of environmental contaminants in animal models is a pivotal factor required for determining their distribution and magnification or of their metabolites as well as evaluating their potential toxic responses. A growing body of experimental evidence presents the skin as one of the main target tissues for PFASs distribution.

The pervasive influences of PFOA have been detected in human communities, animals, and the general environment. Male and female rats, mice, hamsters, and rodents were exposed to a single oral dose of $10 \mathrm{mg} / \mathrm{kg}$ ammonium $\left[{ }^{14} \mathrm{C}\right]$ PFOA by Hundley and colleagues (Hundley et al., 2006). The experimental analysis showed that almost all of the salt administrated was absorbed by the gastrointestinal tract. The recovery of radioactivity indicated that the highest levels of $\left[{ }^{14} \mathrm{C}\right]$ were accumulated in blood, liver, and kidney followed by lung, skin, and testis. It is of note that the elimination of PFOA was reported to be sex and species-dependent (Hundley et al., 2006). Sex-dependent plasma clearance half-life has been observed by Gannon et al., when they studied tissue clearance of [1-14C]-PFHx in rats and mice dosed orally at 2 or $100 \mathrm{mg} / \mathrm{kg}$ (Gannon et al., 2011). $24 \mathrm{~h}$ after a single dose of $100 \mathrm{mg} / \mathrm{kg}$, $\mathrm{PFHx}$ was quantifiable only in the skin as its concentrations were above the limit of quantification (LOQ) levels in the skin of both genders of the two species. Authors deduced from these findings that humans may eliminate PFHx as rapidly as rats and mice.

Bogdanska and colleagues conducted a series of experiments to evaluate the tissue distribution of PFASs in mice (Bogdanska et al., 2011, 2014, 2020). In their earliest study, the tissue distribution of PFOS in C57/BL6 mice was assessed following dietary exposure. Mice were exposed to $0.031 \mathrm{mg} / \mathrm{kg}$ /day of ${ }^{35} \mathrm{~S}$-PFOS for $1-5$ days. The analysis of radioactivity revealed that skin was among the significant compartments containing the largest amount of PFOS. The distribution of PFOS in the skin was homogeneous and dose-dependent (Bogdanska et al., 2011). Perfluorobutanesulfonic acid (PFBS), a four-carbon perfluorobutanesulfonate, was labeled by ${ }^{35} \mathrm{~S}$ and administrated to adult male mice in the same molar concentration of the former study (Bogdanska et al., 2014). Bogdanska et al. (2014) observed that most of ${ }^{35}$ S-PFBS was localized in tissues, including liver, whole bone, blood, skin, and muscle, similar to the observations of PFOS in their previous study (Bogdanska et al., 2011). The tissue concentrations of PFBS, however, were substantially lower than those of its eight-carbon homolog (PFOS). In the most recent study, Bogdanska and colleagues characterized the tissue distribution of ${ }^{14} \mathrm{C}$-PFOA in mice in the same manner that they assessed in the cases of PFOS and PFBS (Bogdanska et al., 2020). They carried out the experiment using two different doses; a high experimental dose $(22 \mathrm{mg} / \mathrm{kg} /$ day $)$ and a lower one $(0.06 \mathrm{mg} / \mathrm{kg} /$ day $)$ similar to those detected in exposed humans. Results revealed that the concentrations of PFOA measured in the blood are in the same range of those reported in humans. Moreover, the distribution profile had a good agreement with the previous findings (Bogdanska et al., 2011, 2014). Interestingly, the level of PFOA in the skin after low-dose exposure was nearly a third of that in the blood. This suggests that PFOA, even at lower doses, leaves the bloodstream and is distributed homogeneously throughout the skin. This suggests that the presence of PFOA in the skin, a tissue with $15-20 \%$ body weight, may play a major part in the wholebody burden.

In agreement, experiments on fish (Goeritz et al., 2013; Falk et al., 2015; Pignotti et al., 2017; Meng et al., 2017), and frog (Cui et al., 2018) corroborated the aforementioned findings and indicated the skin as one of the main areas for localization and recovery of PFASs. Fisheries products are a source of exposure to fluorinated chemicals as shown by the positive correlation between seafood consumption and human exposure to PFASs (Christensen et al., 2017). Market-size rainbow trout (Oncorhynchus mykiss) was exposed to PFHxS, PFOA, PFOS, and PFBS via 
their diet for 28 days. Goertiz et al. identified the skin of fish as one of the main preferential destinations for PFASs deposition (Goeritz et al., 2013) in accordance with findings by another study on this species (Falk et al., 2015). The accumulation of PFASs in the skin was deemed as an intermediate step for subsequent excretion via the skin (Goeritz et al., 2013). However, recent evidence suggests that body surface mucus is responsible for the elimination mechanism of PFOS (Honda et al., 2018). The contribution of edible parts of seafood (skin and muscle) to PFASs exposure is expected to be significant because of their high proportion of the whole weight of the body. In this respect, PFASs uptake by fish dietary intake would reduce with the consumption of fillet without skin (Goeritz et al., 2013).

Remarkable concentrations of PFASs, especially long-chain ones, are magnified in a host of aquatic organisms as a result of bioaccumulation along trophic chains, which are more likely to terminate in humans (Pérez et al., 2014). Despite the ban on the production of PFOS, Pignotti and colleagues detected it as the most abundant perfluoroalkyl compound in a couple of seawater fish species (Pignotti et al., 2017). They evaluated the distribution of several PFASs in the skin and muscles of fishes in different positions of the food chain. Surprisingly, although the concentrations of PFASs were below the method limit of quantification (mLOQ) in water and sediment, a very high amount of PFASs in general, and PFOS in particular, was reported in the fishes ( $\sum$ PFASs range from $63.8 \mathrm{ng} / \mathrm{g}$ ww to $938 \mathrm{ng} / \mathrm{g} \mathrm{ww}$ ), with a relatively more disposition in the skin. It is of relevance that a specific type of PFOS, perfluoroctanesulfonamide, was only detected in predators such as Cyprinus carpio and Silurus glanis, which are at the top of the ecological food chain. Such solid evidence led authors to infer that biomagnification of PFASs continually occurs in aquatic food chains (Pignotti et al., 2017). Similar results have been found in farmed Trachinotus ovatus exposed to PFOS and PFOA. Unlike the abovementioned works, the highest fraction of PFOS was measured in the skin $(18.100 \mathrm{mg} / \mathrm{kg} \mathrm{ww})$ and its distribution declined in the descending order: skin $>$ gill $>$ kidney $>$ liver $>$ flesh (Meng et al., 2017).

There is further evidence confirming the skin as one of the particular spots for the tissue distribution and bioconcentration of PFASs whether alone (Cui et al., 2018; Honda et al., 2018) or in co-exposure with other chemicals (Li et al., 2017). Many amphibians utilize cutaneous gas

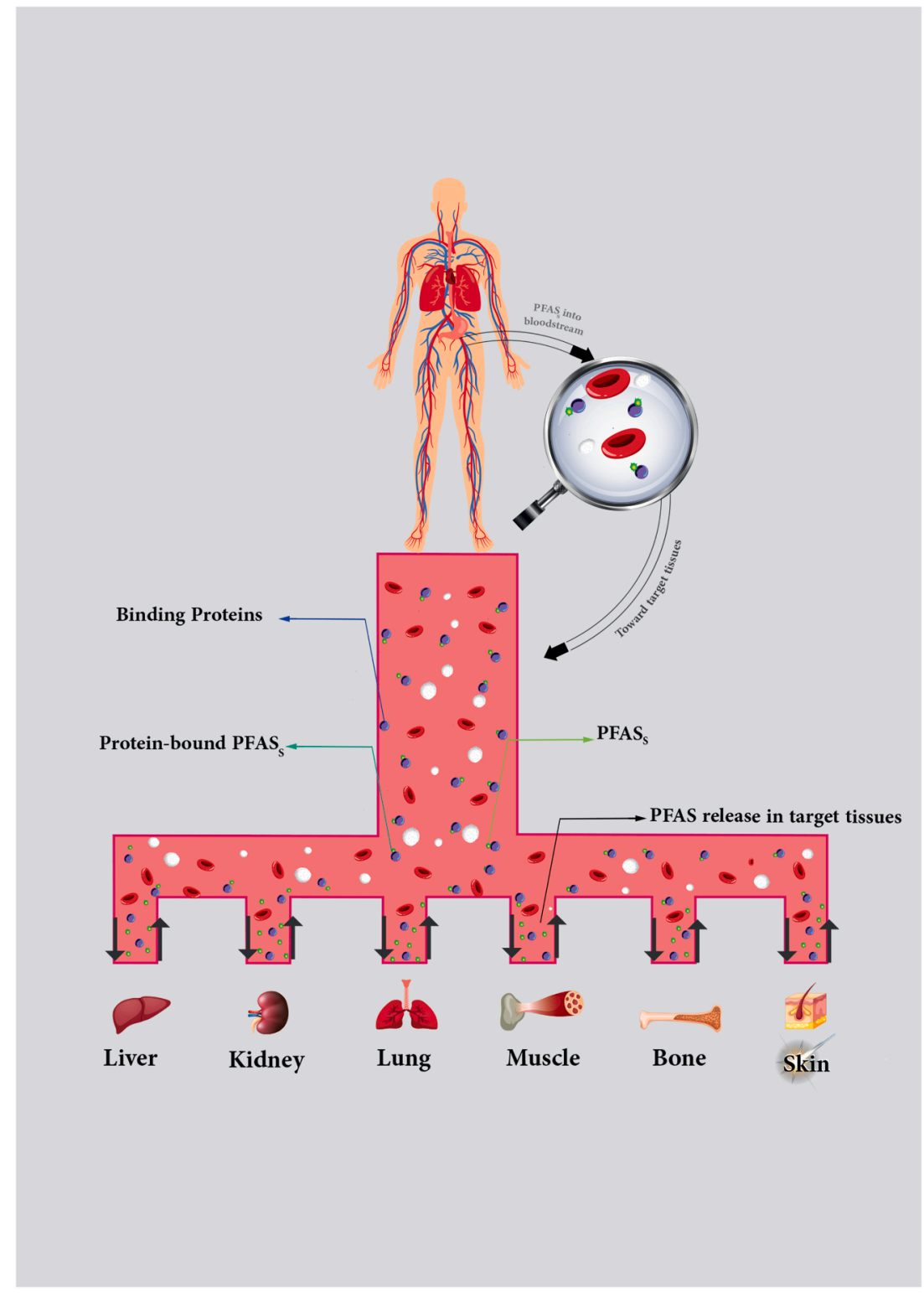

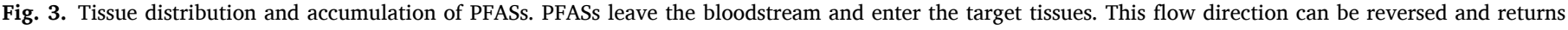
PFASs to the vascular system. Skin is among the main destinations for the accumulation of PFASs after the ingestion of contaminated food, water, and dust. 
exchange as a mode of respiration. Their skin has a high permeability to small molecules affecting the distribution and accumulation of PFASs (Brühl et al., 2013). Recently, it was proven that PFASs bioconcentration in dermally exposed post-metamorphic amphibians depends on the species and the chemical (Abercrombie et al., 2020). For the first time, this influence was examined in frogs collected from Chinese cities with large-scale fluorochemical plants. Cui et al. (2018) observed fairly similar patterns of tissue distribution in frog and fish. More than onethird of the total body burden of PFASs (mainly PFOS and chlorinated polyfluorinated ether sulfonic acid) was found in the skin of males whereas the greatest proportion of the chemicals in females accumulated in the ovary (Cui et al., 2018). Such gender-related differences were also reported in the case of rodents by Hundley et al., (2006). It is expected that the considerable distribution of PFASs in the female sexual organs results in early-life exposure and induces developmental toxicity during pregnancy and infancy.

Investigating the tissue distribution of PFASs shows their capability to leave the bloodstream and enter tissues. The distribution profile of PFASs, according to the findings of the included experimental studies, has been shown in Fig. 3. It is well-known that PFASs exhibit a high binding affinity to serum proteins and fatty acid-binding proteins and this might be the reason why PFASs transfer and accumulate in the target tissues (liver, kidney, lung, muscle, bone, and skin; Goeritz et al., 2013). PFASs have been reported to largely leave the bloodstream and accumulate considerably in tissues in a dose-dependent manner after exposure to high PFASs doses (Bogdanska et al., 2011). The patterns of tissue distribution of contaminants like PFASs in the mentioned animal models indicate general trends and similarities that would be observed in mammals, including probably in humans. As a result, the skin is a potential target tissue for the distribution and accumulation of PFASs through diet, inhalation, and dust dermal exposure.

\subsection{Potential role of PFASs in skin damage}

\subsubsection{Dermal penetration and absorption of PFASs}

Having surfactant properties, PFASs are mostly able to change dermal permeability and break down the skin barrier. Ammonium perfluorooctanoate (APFO) is the ammonium salt of PFOA which can enhance skin penetration in the human and animal skin (Fasano et al., 2005; Kennedy, 1985). APFO was administrated to rats and rabbits to investigate its dermal toxicity. Kennedy Jr (1985) reported that sufficient quantity of APFO had penetrated the skin for the initiation of histomorphologic and physiological changes (Kennedy, 1985). In a similar study, the dermal penetration rate of APFO was evaluated in vitro in rat and human skin. After $48 \mathrm{~h}$, the permeability coefficient for rat and human skin was $3.25 \times 10^{-5} \mathrm{~cm} / \mathrm{h}$ and $9.5 \times 10^{-7} \mathrm{~cm} / \mathrm{h}$, respectively (Fasano et al., 2005). Only a small percentage of applied APFO (1.44\% in rats and $0.048 \%$ in humans) penetrated the skin after $48 \mathrm{~h}$ of exposure. The estimates provided for absorbed dose via dermal exposure demonstrated that the threshold limit value (TLV) may be exceeded under uninterrupted exposure (Fasano et al., 2005). Another study by Franko et al. measured the dermal absorption and penetration of PFOA in mouse and human skin (Franko et al., 2012). The serum level of PFOA significantly increased following dermal exposure in BALB/c mice, which implies its absorption through the skin. In vitro dermal penetration of PFOA indicated that approximately $24 \%$ of applied dose penetrated both full-thickness and epidermis samples of the human skin after $24 \mathrm{~h}$. The total absorbable amount of PFOA (i.e. the sum of the total amount that had penetrated plus the amount originally available in skin samples) for these samples was $69 \%$ and $48 \%$, respectively. In the case of the mice, more than one-third of the administrated dose passed into the skin, and nearly 50\% absorption occurred. It turned out that the ionization state is a determining factor in the absorption degree of PFOA. The less acidity or non-ionized PFOA $(\mathrm{pH}=5.5)$ yields more permeability and easy penetration compared with its ionized form (pH = 2.25) (Franko et al., 2012).
The potential of a chemical for inducing skin irritation and sensitization has a direct correlation with its capability of dermal penetration. Some evidence shows that some PFASs potentially are skin irritant and sensitizer (Kennedy, 1985; Gordon, 2011; Han et al., 2020). Moreover, synthesis and release of cytokines such as interleukin-1 $\alpha$ (IL-1 $\alpha)$ and interleukin-8 (IL-8), two appropriate parameters for screening chemicals for their sensitizing/irritating potential (Coquette et al., 2003), have been reported under exposure to PFASs, strongly suggesting dermal absorption and penetration of these chemicals (Han et al., 2020; Lunardi et al., 2016). Based on the abovementioned research on dermal penetration and absorption of PFASs, the dermal pathway is suggested to be a potential route of exposure to these chemicals.

\subsubsection{Dermal exposure to PFASs and immunotoxic effects}

It is generally known that PFASs, especially longer-chain congeners, have a markedly potential for triggering inflammatory and oxidative reactions. Hue et al. (2020) evaluated the levels of a pro-inflammatory factor (IL-1 $\alpha$ ) and two oxidative stress biomarkers, including malondialdehyde (MDA) and 8-hydroxydeoxyguanosine (8-OHdG) under topically dermal exposure to perfluoroalkyl carboxylic acids (PFCAs) with 5-8 carbons (Han et al., 2020). They used a human skin equivalent (HSE) model entitled EpiDerm Full Thickness (EpiDermFT) composed of constituents such as collagen and human fibroblasts and keratinocytes. This model is used for a variety of experimental analyses on the skin. MDA and 8-OHdG are well-known markers for lipid peroxidation and DNA damage, respectively. The HSE samples were topically exposed to 0.25 and $2.5 \mathrm{mM}$ of the chemicals. In accordance with results from previous studies performed on dermal exposure to different doses of PFOA (Franko et al., 2012), the concentrations under study were noncorrosive. In fact, the concentration, repeat, and period of the exposure were to a great extent similar to those that may occur for the human skin. The measurements revealed that the production of MDA and the release of IL-1 $\alpha$ have significantly increased in tissues treated with $2.5 \mathrm{mM}$ PFASs compared with the control group whereas the level of 8OHdG in the EpiDermFT skin model did not show any meaningful increase (Han et al., 2020). In a study on the immortal human skin keratinocyte (HaCaT) cells, however, exposure to $50 \mu \mathrm{M}$ resulted in a remarkable growth of 8-OHdG-positive staining after a recovery period of 8 days (Peropadre et al., 2018). The discrepancy between these two studies regarding the levels of 8-OHdG could originate from the different human skin models and methodologies applied. Immunological changes in a murine model, including a significant increase of pro-inflammatory cytokines interleukin-1 $\beta$ (IL-1 $\beta$ ), and interleukin-6 (IL-6) have been reported under dermal exposure to $0.5-2 \% \mathrm{w} / \mathrm{v}$, or $12.5-50 \mathrm{mg} / \mathrm{kg} / \mathrm{dose}$ of PFOA (Shane et al., 2020). The positive immunofluorescence staining of IL-6 in HaCaT, a reliable human skin model, was also detected in a significant number of the cells (Peropadre et al., 2018).

Immunotoxicity of PFASs following dermal exposure has been demonstrated by animal studies via the alteration of immune responses and the expression of pro-inflammatory cytokines (Shane et al., 2020; Fairley et al., 2007). In vitro test models (Han et al., 2020; Peropadre et al., 2018) and in-utero studies (Wen et al., 2019a, 2019b) underscore the immunomodulatory role of PFASs. A wealth of evidence suggests that PFOA can act as an immunosuppressive agent. Fairley et al. (2007) demonstrated that dermal exposure to $1-1.5 \%$ PFOA is capable of significant augmentation of IgE antibody and airway hyperreactivity response to ovalbumin, a stimulator of allergic reactions, and probably environmental allergens. This action has been attributed to peroxisome proliferator-activated receptor alpha (PPAR $\alpha$ ), a ligand-activated transcription factor that plays a pivotal role in the skin homeostasis. This nuclear factor regulates inflammatory responses in the skin, and it is expressed in keratinocytes, T-lymphocytes, and macrophages (Dubrac and Schmuth, 2011). PPAR $\alpha$ agonists are applied in pharmaceuticals to treat inflammatory skin diseases (Hawkins et al., 2010). With this antiinflammatory role of PPAR $\alpha$ in mind, any environmental factor with the capability of decreasing the expression of PPAR $\alpha$ nuclear factor can 
disrupt skin homeostasis and jeopardize skin integrity (Shane et al., 2020). Nuclear factor of kappa light polypeptide gene enhancer in Bcells 1 (NFKB1) is a transcription factor that similar to PPAR $\alpha$ fights against inflammation, senescence, and carcinogenesis (Cartwright et al., 2016). Down-regulation of these two anti-inflammatory proteins has been reported in the murine model administrated dermally to PFOA during 14-days (Shane et al., 2020).

Exposure to PFASs exacerbates allergic responses (Fairley et al., 2007) and has been associated with atopic diseases (AD) (Wen et al., 2019a; Dubrac and Schmuth, 2011). Wen and colleagues shed light on the association between PFOA exposure and atopic dermatitis (Wen et al., 2019a, 2019b). AD, also known as atopic eczema, is the most common type of skin disease that typically starts at childhood and is characterized by the occurrence of a long-term skin inflammation (TUVW, 2016). Through a 5-year follow-up study, prenatal exposure to PFOA was positively associated with earlier onset of AD since higher prenatal PFOA exposure enhanced the risk of AD development (Wen et al., 2019b). The combined effect of exposure to PFOA and glutathione S-transferase (GST) T1/M1 genotype on childhood AD has been explained using a 2-year follow-up birth cohort study in Taiwan (Wen et al., 2019a). GST is an enzyme catalyzing the conjugation of glutathione (a powerful antioxidant) to xenobiotics such as PFASs in order to detoxify these. Therefore, it is expected that individuals with null types of GST may be less equipped to overcome the oxidative situation due to having dysfunctioned enzymatic activity. Subsequently, reactive oxygen species (ROS) overproduction can initiate and elicit the activation of inflammatory cells and the expression of pro-inflammatory cytokines. It was revealed that the gene-environment interaction in the form of inutero PFOA exposure and GSTT1/M1 null phenotype might synergistically heighten the risk of AD in children (Wen et al., 2019a). Likewise, prenatal exposure to other PFASs such as PFHxS and perfluorodecanoic acid (PFDA) substantially increases the risk of AD in female children (Chen et al., 2018). Overall, PFASs have the potential to elicit inflammation and oxidative responses in the skin leading to skin inflammation and oxidative damage.

\subsubsection{DNA damage under exposure to PFASs}

Telomeres in mammal cells consist of a non-coding region of DNA with repetitive sequences of $5^{\prime}$-TTAGGG- $3^{\prime}$ found at each end of eukaryotic chromosomes. Steady telomere shortening probably acts as a mitotic clock that regulates DNA replication and restricts cell proliferation. Typically, they are shortened over the cell life as every cell division moves telomeres further toward a critical length where programming cell self-destruction occurs (Møller et al., 2018). Therefore, it is speculated that telomere shortening would be a mechanism for preventing carcinogenesis through the induction of cellular aging and senescence. Some epidemiological studies have associated the length of this single-stranded overhang with environmental and occupational pollutants (Zhang et al., 2013). On top of that, toxicological investigations have considered telomere shortening and decreasing telomerase activity as hallmarks of premature cellular senescence induced by exposures to halogenated contaminants such as PFASs (Møller et al., 2018; Liu et al., 2018). Nevertheless, a few numbers of studies reported the positive association between exposure to chemicals and telomere elongation (Zhang et al., 2013; Blévin et al., 2017). The elongation of telomeres takes place in proliferating cells by the mediatory role of the telomerase enzyme adding tandem repeats of $5^{\prime}$-TTAGGG-3' to DNA (Zhang et al., 2013). Increasing telomere length under exposure to chemicals has been ascribed to environmental and nutritional conditions, which carry more weight by comparison (Blévin et al., 2017); thus, such environmental conditions stimulate regulated telomerase activity which is growth-modulated. It is worth mentioning that telomerase is detected in somatic tissues with high proliferation capacity (e. g. skin, intestines, and bone marrow) and proliferating normal stem-like cells such as human $\mathrm{T}$ cells. In fact, not only telomerase is expressed in tumor tissues, but also accumulating evidence suggests that it is expressed in a variety of normal human tissues in a controlled level (Shay and Wright, 2019).

Inflammatory responses and oxidative stress are two effective factors playing a destructive role in the regulation of telomere length and telomerase activity. On the other hand, the increment of free radicals as well as telomere shortening rate are key elements in the aging process. ROS may attack DNA nucleobases and form products that substantially put telomeres in danger. Although the exact influence of ROS on telomere is not fully understood, it is stated that both chronic and acute exposure to oxidizing free radicals may give rise to DNA strand breaks leading to telomere loss (Møller et al., 2018). Measuring concentrations of PFASs, ROS, and leukocyte telomere length in umbilical cord blood of 581 newborns have demonstrated that telomere length has an inverse association with the level of ROS and concentration of PFASs, especially PFOS and PFDA in female newborns (Liu et al., 2018).

Likewise, in the case of skin cells, telomeres are of high importance in their life cycle and play a part in skin aging. Skin is susceptible to premature telomere shortening through agents such as inflammatory mediators, free radicals, and chemicals which interfere in the cell proliferation. DNA damage, cell cycle arrest, and senescence of skin cells can be triggered by telomere attrition (Buckingham and Klingelhutz, 2011). HaCaT, a cell line from adult human skin, is a suitable candidate to assess dermal toxicity and irritancy (van de Sandt et al., 1999). Exposure of $\mathrm{HaCaT}$ to bioaccumulative and degradable-resistant organic pollutants shortens the telomere length and declines telomerase activity (Senthilkumar et al., 2011; Senthilkumar et al., 2012). Peropandre et al. (2018) evaluated cytotoxic indices such as cell proliferation, DNA damage, oxidative stress, and cell senescence in epidermal HaCaT keratinocytes under exposure to PFOA. The proliferation ability of HaCaT cells was impaired during $24 \mathrm{~h}$ after a single dose of the chemical and did not restore within the recovery period. Treatment of HaCaT cells with $50 \mu \mathrm{m}$ of PFOA for $24 \mathrm{~h}$ resulted in a significant increase of nuclear $\gamma$-H2AX foci, a marker of DNA double-strand breaks (DSBs). Moreover, significant positive staining for acidic $\beta$-galactosidase has been reported (Peropadre et al., 2018). Senescence-associated $\beta$-galactosidase (SA$\beta$-gal) activity and the number of $\gamma$-H2AX foci are well-known biomarkers involved in the development of senescence-associated secretory phenotype (Herbig et al., 2006; Debacq-Chainiaux et al., 2009).

\subsection{Biological mechanisms involved in PFASs exposure and premature skin aging}

During natural skin aging, the skin ability to heal and rejuvenate diminishes, dermal collagen and elastin are fragmented, and undesirable aesthetic signs like uneven tone, wrinkling, and loss of elasticity, and thinning develop (Farage et al., 2007). Both intrinsic and extrinsic skin aging are accompanied by underlying mechanisms such as reduction of skin integrity, impairment of immune responses, the increment of oxidative stress, degradation of extracellular matrix (ECM), dramatic up-regulation of matrix metalloproteinases (MMPs), disruption of vitamin D endocrine system, and signaling of the mitogen activated protein kinase (MAPK) pathway (Elsner et al., 2009; Krutmann et al., 2017). Chronic dermal exposure to PFASs would occur directly via consumer products and indirectly by their bioaccumulation in the skin (Figs. 2 and 3). The role of PFASs exposure in skin aging is summarized in Fig. 4 and discussed in detailed below.

\subsubsection{Production of free radicals and reactive oxidative species}

It is generally acknowledged that PFASs induce the overproduction of free radicals, including ROS, resulting in a significant decrease in total antioxidant capacity, which in turn puts target tissues in an oxidative state (Wielsøe et al., 2015). Based on the experimental studies on a human skin model, exposure to PFASs initiates oxidative stress through the production of MDA, with values 1.3-2.1 times higher than the control group, with the exception of those treated with perfluorohexanoic acid (Han et al., 2020). The increase of MDA levels has 


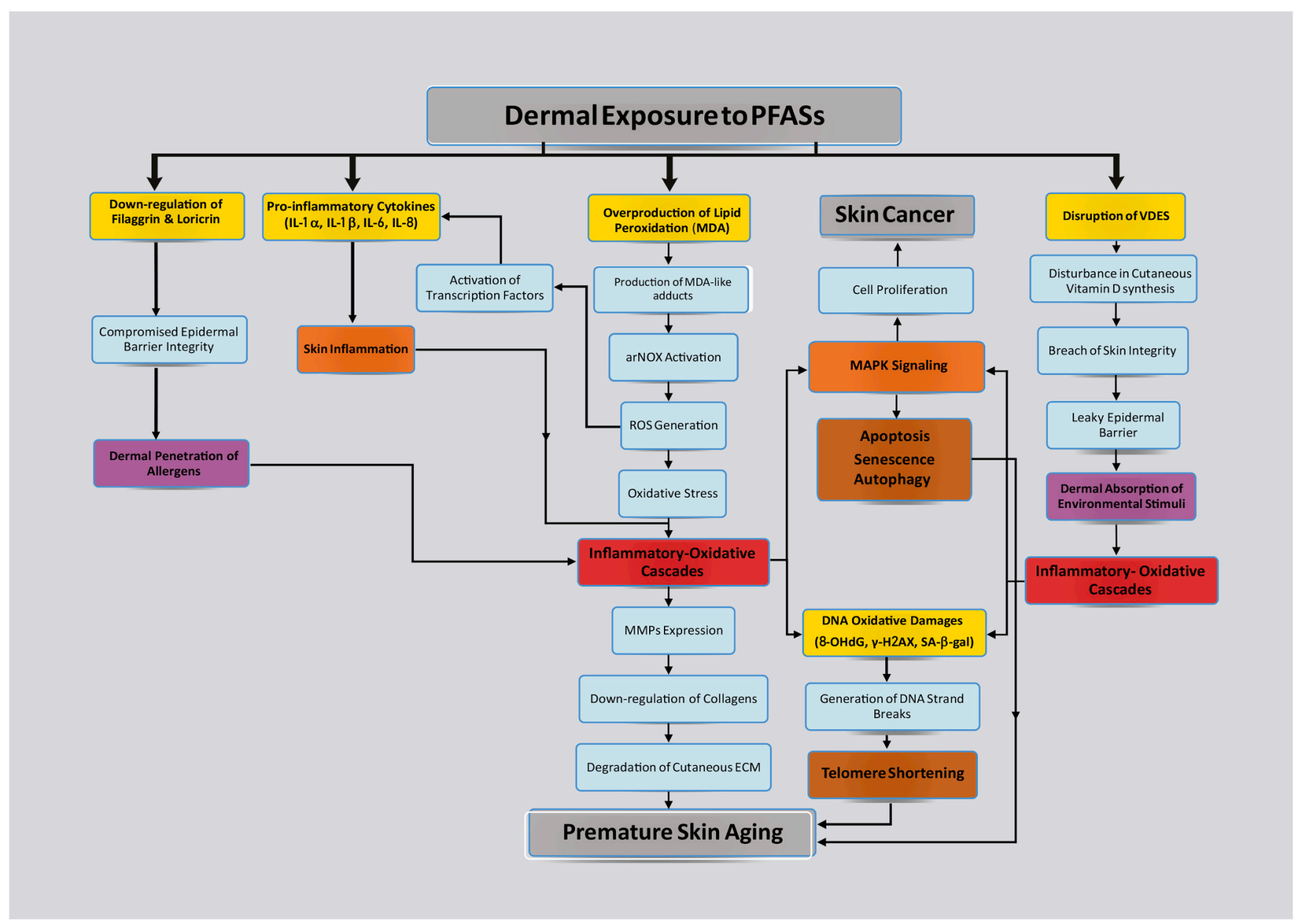

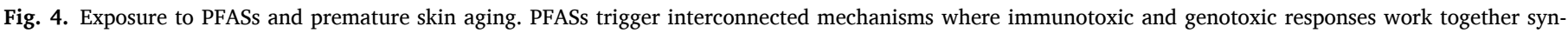
ergistically and establish inflammatory-oxidative cascades. Reinforcing these reactions by compromised skin integrity results in premature skin aging.

been reported in tissues of animal models exposed to PFOA (Yang et al., 2014; Liu et al., 2007). MDA is one of the most prominent products of peroxidation of polyunsaturated fatty acids which reacts with macromolecules and forms protein and DNA adducts, major contributors to the aging process (Popoola et al., 2015; Całyniuk et al., 2016). Recently, a novel mechanism of aging explained how MDA could engage in skin senescence. The mechanism is activated by age-related NADH oxidase (arNOX) and propagates ROS production at the cell surface to surrounding cells, similar to the mechanisms of natural skin aging (Morre and Morre, 2014). It was shown that the formation of MDA-like adducts involved in the oxidation of lipoproteins correlates with arNOX (Morré and Morré, 2014). Going into the depth, individuals with higher activity of arNOX suffers from skin characteristics that make them appear older than their chronological ages. Accordingly, MDA is capable of strengthening the production and dissemination of free radicals in the skin by the help of this ROS generator (Peres et al., 2011). Fig. 4 depicts the chain of events through which the generation of oxidative agents contributes to the process of skin aging.

\subsubsection{Production of pro-inflammatory cytokines}

Pro-inflammatory cytokines play an indispensable part in skin aging process as an imbalanced production of these proteins produces discernible changes in the skin appearance (Borg et al., 2013). The skin is an immunologically active organ, and therefore would modulate and synchronize pro- and anti-inflammatory responses (Farage et al., 2009). PFASs have the potential to considerably lower the levels of leukocytes, lymphocytes, and neutrophils in the skin (Shane et al., 2020) and to increase the mRNA levels of pro-inflammatory cytokines such as IL-1, IL6 , and tumor necrosis factor-alpha (TNF- $\alpha$ ) in different tissues (Shane et al., 2020; Qazi et al., 2009; Dong et al., 2011). The age-associated cutaneous pigmentation may originate from the production of endothelin-1 (ET-1) by keratinocytes triggered via the increased secretion of IL-1 $\alpha$ (Okazaki, 2009). Under normal circumstances, IL- $1 \alpha$ is stored in keratinocytes but it is released after membrane breakdown or cell damages. The levels of IL-1 $\alpha$ were markedly enhanced in EpiDermFT treated with $2.5 \mathrm{mM}$ PFOA suggesting that PFOA can induce keratinocyte membrane perturbation (Han et al., 2020). Besides, significant expression of cytokines such as IL-1 $\beta$ (Shane et al., 2020) and IL-6 (Peropadre et al., 2018) was reported in the skin after dermal exposure to PFOA. IL-1 and IL- 6 stimulate the expression of collagendegrading enzymes such as MMPs leading to the decreased production of collagen and disruption in collagen homeostasis (Maggio et al., 2006; Baugé et al., 2007). IL-1 $\alpha$, IL-1 $\beta$, IL-6, IL-8 are well-known components of the senescence-associated secretory phenotype as they are upregulated in aged skin leading to skin inflammation (Chung et al., 2008; Coppé et al., 2010). Transcriptomic analysis of bottlenose dolphin skin biopsies cultured and treated with PFOA revealed variations in the expression of genes involved in the immune system. For instance, IL-8, an inflammatory cytokine engaged in neutrophil activation, showed a 6.8 -fold increase (Lunardi et al., 2016). The overproduction of oxidative agents activates NF- $\mathrm{\kappa B}$ leading to the up-regulation of IL-1, IL- 6 , and IL-8 (Maggio et al., 2006). Shane et al. (2020) reported that exposure to PFOA has also been associated with the increase of gene expression of $\mathrm{T}$ helper cell type 2 (Th2) skewing cytokines, involved in allergic reactions 
and atopic skin conditions (Berger, 2000), and with a reduction of gene expression of peroxisome proliferator-activated receptor alpha involved in the anti-inflammatory response (Dubrac and Schmuth, 2011). The oxidative stress produces the expression of pro-inflammatory factors leading to further dermal inflammation. Based on the reciprocal connection between oxidative stress and inflammation; and on the occurrence of these conditions in human skin models under chronic exposure to PFASs on the other hand, it can be hypothesized that the formation of inflammatory-oxidative cascades upon PFASs exposure may be plausible mechanism producing skin aging (Fig. 4). This mechanism would be consistent with observations from other exogenous factors involved in the skin aging process (Ahuja et al., 2017).

\subsubsection{Production of DNA oxidative damage}

As summarized in Fig. 4, increased secretion of pro-inflammatory molecules and free radicals potentiate skin aging by their structurally detrimental impacts on DNA, including but not limited to singlestranded and double-stranded DNA breaks, telomere shortening, and damages to the DNA repair system (Victorelli and Passos, 2020; Ahsanuddin et al., 2016). In a narrow-age cohort of older adults, particular skin aging features such as wrinkles, facial sagging, and pigmented spots were positively associated with high concentrations of 8-OHdG, a critical biomarker of oxidative stress (Allerhand et al., 2011). PFASs are capable of inducing oxidative stress and oxidative DNA damage via the production of 8-OHdG (Peropadre et al., 2018; Zhao et al., 2019; Yahia et al., 2016). For example, exposure of epidermal HaCaT keratinocytes to $50 \mu \mathrm{m}$ PFOA for $24 \mathrm{~h}$ resulted in the moderate increase of 8 -OHdG concentrations observed 8 days after exposure cessation (Peropadre et al., 2018). On the other hand, the excessive formation of oxidative stress (8-OHdG) may in turn accelerate telomere shortening rate (Fouquerel et al., 2019). Likewise, premature telomere shortening would be another possible mechanism through which 8-OHdG may contribute to skin aging.

Phosphorylation of $\mathrm{H} 2 \mathrm{AX}$, the minor histone $\mathrm{H} 2 \mathrm{~A}$ variant, produces $\gamma$-H2AX which is another hallmark of DNA damages. It is taken into consideration as a cellular reaction to DSBs, one of the worse forms of DNA lesions (Redon et al., 2002; Mah et al., 2010). Formation and accumulation of irreparable DSBs, indicated by the persistent presence of $\gamma$-H2AX foci, has an essential role in starting cellular senescence (Fumagalli et al., 2012). A significant increase in $\gamma$-H2AX foci under exposure to PFOS and PFOA represents another mechanistic pathway in which PFASs can exert genotoxic impacts mediated by oxidative stress (Peropadre et al., 2018; Wang et al., 2015). The possibility of DSBs induction in HaCaT cells treated with $50 \mu \mathrm{M}$ PFOA was indirectly assessed by using $\gamma$-H2AX immunofluorescence. PFOA exposure in epidermal HaCaT keratinocytes has displayed a marked increase of $\gamma$-H2AX-positive cells and significant positive staining for acidic $\beta$-galactosidase (SA$\beta$-gal) (Peropadre et al., 2018). Notably, telomere shortening strongly correlates with SA- $\beta$-gal and the number of $\gamma$-H2AX foci (Bernadotte et al., 2016). These observations in the lower layers of the HaCaT cells are associated with the features developing senescence-associated secretory phenotype. As a result, mild exposure to PFOA has the potential to induce DNA damages and senescence in dermal HaCaT cells (Peropadre et al., 2018). In summary, the evidence suggests that oxidative DNA damages induced under exposure to PFASs produces 8OHdG, $\gamma$-H2AX as well as activates $\beta$-galactosidase providing a plausible mechanism by which dermal exposure to PFASs may increase telomere shortening and consequently produce premature skin aging.

\subsubsection{Disruption of vitamin $D$ endocrine system}

The skin acts both as the main generator and as a target tissue for the biologically active metabolites of vitamin $\mathrm{D}_{3}$, especially 1,25 -dioxyvitamin $\mathrm{D}\left[1,25(\mathrm{OH})_{2} \mathrm{D}\right]$. The latest evidence suggests that the vitamin $\mathrm{D}$ endocrine system (VDES) exerts considerable influences over the aging process in different tissues, including the skin (Reichrath, 2012; Chang et al., 2010). Mechanistically speaking, VDES contributes to skin health via several biochemical pathways. These are the down-regulation of genes responsible for oxidative damage, inflammation, and cellular aging, the preservation of telomere biology, the inhibition of UV-Binduced cleavage of the Poly-(ADP-Ribose)-Polymerase, and the induction of synthesis of metallothionein, an antioxidative protein (Reichrath, 2012). In vivo studies suggest the modulatory role of vitamin D and its analogs in the biology of keratinocytes and melanocytes of the skin (Piérard-Franchimont et al., 2007). Through a mechanistic insight, Mousavi et al. (2019) meticulously elucidated how endocrine-disrupting chemicals (EDCs) disturb VDES and trigger the insufficient and deficient serum levels of biologically active forms of vitamin D. Since PFASs are also categorized as EDCs, it is expected that these persistent chemicals alter circulating levels of active metabolites of vitamin D. Limited epidemiological evidence exists to examine this association (Pearl et al., 2018; Etzel et al., 2019). A cross-sectional analysis investigated the relationship between four abundant PFASs, including perfluorononanoic acid (PFNA), PFOS, PFHxS, and PFOA, with the serum concentrations of 25-hydroxyvitamin D [25(OH)D] (Etzel et al., 2019). PFASs were detected in $98 \%$ of participants' samples and two different patterns were observed regarding the correlation between PFASs exposure and serum 25(OH)D levels. In the adjusted model, each 2-fold increment of PFOS was associated with lower levels of $25(\mathrm{OH}) \mathrm{D}(0.9 \mathrm{nmol} / \mathrm{L}, 95 \% \mathrm{CI}: 0.2$, 1.5). The association was statistically significant in the case of PFHxS ( $0.8 \mathrm{nmol} / \mathrm{L}, 95 \% \mathrm{CI}$ : 0.3, 1.3 per each 2 -fold increment), while no association was observed for PFOA or PFNA (Etzel et al., 2019). In another study by Pearl et al. (2018), 2-(N-Ethyl-perfluorooctane sulfonamide) acetate (Et-PFOSA-AcOH) was inversely associated with the level of 25 $(\mathrm{OH}) \mathrm{D}$ measured in serum of pregnant women. Concentrations of serum Et-PFOSA-AcOH were associated with increased odds of 25(OH)D insufficiency (OR $=1.3,95 \%$ CI 1.0, $1.7 \mathrm{per} \mathrm{nmol} / \mathrm{L}$ ), whereas elevated maternal serum concentration of PFNA was associated with decreased odds of vitamin D insufficiency ( $\mathrm{OR}=0.6,95 \% \mathrm{CI} 0.4,0.9$ ) (Pearl et al., 2018). Although there is inconsistency in the direction of the observed results, both studies report alterations in the serum levels of vitamin D triggered by PFASs exposure. These results are of interest as VDES plays an underlying part in the aging process through signaling pathways in which tumor protein p53 and fibroblast growth factor 23 (FGF-23) are involved in mechanisms resulting in damages to DNA and telomere shortening (Reichrath, 2012; Pusceddu et al., 2015). Due to the capability of PFASs disturbing VDES and the association of vitamin D level with the aging process, PFASs-originated disruption of normovitaminosis D could potentially accelerate premature skin aging, which in turn could perturb the synthesis of cutaneous vitamin D (Fig. 4).

Sufficient levels of vitamin D decelerates skin aging through the prevention of UV-derived skin and DNA damage, the increase of collagen levels, and the maintenance of skin integrity (Raj et al., 2017). Skin integrity plays a determining role in maintaining its homeostasis and normal functions. Skin integrity is associated with factors including vitamin $\mathrm{D}$ concentration and maintenance of acidic $\mathrm{pH}$ (Farage et al., 2013). Accordingly, inasmuch as PFASs exposure could interfere VDES, it could deteriorate skin integrity due to decreased levels of vitamin D. Moreover, a breach in the epidermal barrier facilitates the dermal absorption of environmental chemicals as well as the skin penetration by allergens (Hughes, 2017). Consequently, environmental insults, including pathogenic microorganisms, pollutants, toxic chemicals, and UV radiation could penetrate into the damaged skin potentially leading to persistent inflammatory-oxidative cascades. Such a situation could represent a negative loop between the quality and quantity of vitamin D and skin integrity potentially worsening skin conditions and accelerating skin aging.

\subsubsection{Downregulation of components that contribute to skin integrity}

Aging imposes profound impacts on the epithelial barrier's structures and functions (Parrish, 2017). In aging skin, the permeability barrier and its structural integrity are significantly disrupted as a result of the activation of MMPs and subsequent ECM degradation (Farage 
et al., 2014). Although not in cutaneous cells, tight junction disruption, barrier integrity breakdown, and MMPs upregulation have been reported as a response to PFASs exposure (Yu et al., 2020; Qiu et al., 2016; Zhang et al., 2014; Saejia et al., 2019). Moreover, the decrease of overall thickness in the EpiDermFT skin model caused by exposure to $2.5 \mathrm{mM}$ PFOA may be partially interpreted as the loss of ECM which can in turn be attributed to the activation of epidermal MMPs (Han et al., 2020). The metabolism of collagen, one of the most principal proteins of dermal ECM, is vital for maintaining skin barrier function and integrity (Genovese and Sibilla, 2015). Exposure of epidermal cell cultures of bottlenose dolphins to $13 \mathrm{ppm}$ PFOS caused alterations in the expression of genes associated with the decrease of cell proliferation and activation of stress responses. Most importantly, significantly decreased expression of collagen type XII has been observed after 1 or $25 \mathrm{~h}$ of exposure (Mollenhauer et al., 2009). This type of collagen is expressed in dense connective tissues primarily composed of collagen type I. Collagen XII takes part in tissue remodeling and the organization of ECM architecture of the skin whilst mutations of genes expressing collagen XII leads to a disruption of the matrix structure (Reichenberger et al., 2000). No sign of the decreased level of collagen type I was observed in the human skin model exposed to $2.5 \mathrm{mM}$ of several PFASs (Han et al., 2020). The deductive reasoning of Han and colleagues puts an emphasis on the possible role of PFASs on skin senescence. However, two major components of the skin integrity (collagen type I and filaggrin) which are affected in the aging process, were strongly stained (i.e. immunohistochemical staining technique) throughout the dermal layer in all groups treated with the examined PFASs. Such a seemingly dichotomy between normal filaggrin expression and other microscopic observations (epidermal vacuolization, necrotic tissues, and decreasing skin thickness) could be the result of insufficient exposure duration, or due to the lack of an observable effect. In addition to the exposure period, collagen type I and filaggrin may require slightly higher concentrations of PFOA to be down-regulated in a short-time exposure. Hence, evaluating the chronic effects of PFASs on the constituents of dermal ECM is one area that requires further research.

Filaggrin is a structural filament-associated protein binding to keratin fibers in epithelial cells and plays an essential role in the formation and maintenance of the epidermal skin barrier, particularly stratum corneum (SC), the outmost layer of the skin (Sandilands et al., 2009). It fulfills many significant functions in the skin, including the regulation and maintenance of skin hydration, skin $\mathrm{pH}, \mathrm{SC}$ barrier integrity, and probably skin buffering capacity (Rinnerthaler et al., 2013). Hence, the down-regulation of filaggrin in aged skin participates in the pathogenesis of inflammatory skin diseases, breaking down the epidermal barrier integrity, and increasing water loss (Sandilands et al., 2009). Recently, two studies measured filaggrin expression in two different in vitro human skin models following exposure to two different dosages of PFASs (Han et al., 2020; Shane et al., 2020). Dermal administration of $0.5-2 \%$ PFOA in a murine model led to a decrease of filaggrin and loricrin, both of which are structural proteins found in the cornified cell envelope (CE) in epidermal cells (Shane et al., 2020). Importantly, CE is the frontline defense against environmental stimuli and, therefore, it is effectively involved in the skin aging process where its protein composition changes drastically (Rinnerthaler et al., 2013). It is of note that the decreased levels of PPAR $\alpha$ and NFKB1 under PFOA exposure were concomitant with the declined levels of filaggrin and loricrin. Therefore, PFOA could potentially disturb the immunological functions of the skin and damage the skin barrier. The reduced amount of filaggrin and loricrin is associated with poor skin integrity, and it is a hallmark of the rebuilt of CE observed in aging skin as well as in a couple of skin disorders such as AD, ichthyosis, etc (Streubel et al., 2017). On the contrary, the study by Han and colleagues (2020) did not reported reduced expression of filaggrin and collagen type 1 in HSE treated with $0.1 \%$ of PFOA and short-chain PFCAs (Han et al., 2020). It should be noted that neither the concentrations of PFASs used nor the exposure duration in the HSE model was similar to that applied in the murine model.
Nevertheless, the decrease of overall skin thickness, necrotic keratinocytes and fibroblasts, and intracytoplasmic vacuolation in the epidermal granulosum layer were observed in PFOA-treated tissues (Han et al., 2020). These structural observations are among the visual phenotype and degenerative changes of skin cells undergoing aging in vitro (Farage et al., 2017; Rattan, 2017).

\subsubsection{Signaling of the mitogen activated protein kinase (MAPK) pathway}

Elevated ROS was associated with the activation of the MAPK pathway downstream of $\mathrm{TNF} \alpha$, including the p38 pathway responsible for the ROS-mediated cell apoptosis (Yu et al., 2015) and in autophagy regulation (Gu et al., 2020). Lu et al. (2016) reported that PFOA exposure induced p38 MAPK signaling in the blood-testis barrier. In turn, p38 MAPK signaling is related to the induction of apoptosis through a p53dependent mechanism (Ge et al., 2016). p53 is a tumor suppressor protein that plays vital roles in the regulation of cellular processes, such as modulating the cell cycle and the inducing apoptosis (Harris and Levine, 2005). On the other hand, the activated form of p38 MAPK, the phosphorylation of p38 MAPK (P-p38 MAPK) has been reported to be elevated in cancerous cells (e.g. breast cancer cells) (Esteva et al., 2004).

P-p38 MAPK pathway can be signaled in response to external stressors, such as ultraviolet irradiation and chemicals (Dérijard et al., 1994). Lu et al. (2016) reported a dose-dependent increase of the p-p38 MAPK/p38 MAPK ratio in the testes of male mice after PFOA administration, with the group exposed to $20-\mathrm{mg} / \mathrm{kg} / \mathrm{d}$ PFOA being statistically significantly different from the control group. In addition, the P-p38 MAPK pathway can also be activated in response to endogenous stressors such as growth factors and cytokines, which are overexpressed after exposure to PFOS (Peropadre et al., 2018; Shane et al., 2020). The p38 MAPK plays a dual role as a regulator of cell death. It can mediate cell survival but also cell death depending on the type of stimulus and type of cell (Koul et al., 2013). Therefore, the activation of the p38 MAPK pathway upon exposure to PFOS could result in skin cell apoptosis, senescence, and autophagy leading to skin aging, or alternatively, could promote cell proliferation potentially leading to skin cancer.

\section{Future research}

To ascertain the role of direct dermal exposure and/or indirect exposure through skin bioaccumulation of PFASs in accelerated skin aging, the following research is recommended:

- Investigation of PFASs exposure on the expression, release, and activation of antioxidant enzymes (e.g. superoxide dismutases), ROSgenerator enzymes (e.g. NADH oxidases, nitric oxide synthases), and the antioxidant reservoir of the skin tissue.

- The contribution of PFASs in the formation of inflammatoryoxidative cascades in skin tissue, especially in connection with the mediatory role of transcription factors such as NF- $\mathrm{BB}, \operatorname{PPAR} \alpha$, and activator protein 1 (AP-1).

- Induction of DNA damage biomarkers, e.g. examining the formation of DNA adducts under dermal exposure to PFASs.

- The associations between PFASs exposure with telomerase activity and telomere shortening rate in a population chronically exposed to PFASs.

- The possible relationship between exposure to PFASs and the disruption of VDES functions, and their effect on skin senescence.

- Expression and activation of enzymes involved in the degradation of components that contribute to epidermal integrity, MMPs in particular, under long-term dermal exposure to PFASs.

- Elucidation of the conditions in which exposure to PFASs activates MAPK signaling pathway leading to apoptosis, autophagy, and senescence of skin cells. 


\section{Conclusion}

The existing evidence suggests that skin absorption of PFASs through dermal contact may be an important route of exposure to these chemicals in humans. On the other hand, PFASs intake by other routes may eventually increase dermal exposure via tissue bio-distribution leading to PFASs bioaccumulation in the skin. Additionally, the presence of PFASs in consumer and cosmetic products, combined with their daily close contact with the skin could render humans readily susceptible to dermal absorption. Therefore, chronic low-dose dermal exposure to PFASs could occur in the human population, representing another important route of exposure to these chemicals. Further research requires a holistic approach to investigate the importance of PFASs exposure across several routes of exposure.

To the best of our knowledge, PFASs exposure can exert detrimental effect on skin health and could accelerate skin aging. The plausible biological mechanisms through which PFASs could accelerate skin aging include oxidative DNA damage, overproduction of ROS, up-regulation of pro-inflammatory cytokines, the disruption of VDES, the downregulation of genes involved in establishing epidermal integrity and cohesion, and signaling of the MAPK pathway. These events may participate in the formation of inflammatory-oxidative cascades resulting in the activation of MMPs, degradation of ECM, and telomere shortening; all of which accelerate the process of skin aging. Further research is required to evaluate the plausibility, importance and synergic effects of the aforementioned mechanisms on skin damage associated with PFASs exposure.

\section{CRediT authorship contribution statement}

Sayed Esmaeil Mousavi: Conceptualization, Methodology, Investigation, Resources, Writing - original draft, Visualization, Project administration, Writing - review \& editing. Juana Maria DelgadoSaborit: Validation, Writing - review \& editing. Lode Godderis: Validation, Writing - review \& editing.

\section{Declaration of Competing Interest}

The authors declare that they have no known competing financial interests or personal relationships that could have appeared to influence the work reported in this paper.

\section{Appendix A. Supporting information}

Supplementary data associated with this article can be found in the online version at doi:10.1016/j.jhazmat.2020.124256.

\section{References}

Abercrombie, S.A., Perre, C., Iacchetta, M., Flynn, R.W., Sepúlveda, M.S., Lee, L.S. Hoverman, J.T., 2020. Amphibian sublethal exposure to perfluoroalkyl substances. Environ. Toxicol. Chem., etc.4711

Ahsanuddin S., Lam M., Baron E.D., 2016 Skin aging and oxidative stress.

Ahuja, A., Singh, N., Gupta, P., Mishra, S., Rani, V., 2017. Influence of exogenous factor on skin aging. In: Farage, M.A., Miller, K.W., Maibach, H.I. (Eds.), Textbook of Aging Skin. Springer, Berlin, Heidelberg, pp. 563-577.

Allerhand, M., Ting Ooi, E., Starr, R.J., Alcorn, M., Penke, L., Drost, E., MacNee, W., Deary, I.J., Starr, J.M., 2011. Skin ageing and oxidative stress in a narrow-age cohort of older adults. Eur. Geriatr. Med. 2 (3), 140-144.

ATSDR (AfTSaDR), 2018. Toxicological profile for perfluoroalkyls draft for public comment. https://www.atsdr.cdc.gov/toxprofiles/tp200.pdf.

Barzen-Hanson, K.A., Roberts, S.C., Choyke, S., Oetjen, K., McAlees, A., Riddell, N., McCrindle, R., Ferguson, P.L., Higgins, C.P., Field, J.A., 2017. Discovery of 40 classes of per-and polyfluoroalkyl substances in historical aqueous film-forming foams (AFFFs) and AFFF-impacted groundwater. Environ. Sci. Technol. 51 (4), 2047-2057.

Baugé, C., Legendre, F., Leclercq, S., Elissalde, J.M., Pujol, J.P., Galéra, P.,

Boumédiene, K., 2007. Interleukin-1 $\beta$ impairment of transforming growth factor $\beta 1$ signaling by DOWN-REGULATION of transforming growth factor $\beta$ receptor type II and up-regulation of Smad7 in human articular chondrocytes. Arthritis Rheum. Off. J. Am. Coll. Rheumatol. 56 (9), 3020-3032.

Berger, A., 2000. Th1 and Th2 responses: what are they? BMJ 321 (7258), 424.
Bernadotte, A., Mikhelson, V.M., Spivak, I.M., 2016. Markers of cellular senescence. Telomere shortening as a marker of cellular senescence. Aging (Albany NY) 8 (1), $3-11$.

Blévin, P., Angelier, F., Tartu, S., Bustamante, P., Herzke, D., Moe, B., Bech, C., Gabrielsen, G.W., Bustnes, J.O., Chastel, O., 2017. Perfluorinated substances and telomeres in an Arctic seabird: cross-sectional and longitudinal approaches. Environ. Pollut. 230, 360-367.

Bogdanska, J., Borg, D., Sundström, M., Bergström, U., Halldin, K., Abedi-Valugerdi, M., Bergman, ^., Nelson, B., DePierre, J., Nobel, S., 2011. Tissue distribution of 35Slabelled perfluorooctane sulfonate in adult mice after oral exposure to a low environmentally relevant dose or a high experimental dose. Toxicology 284 (1-3), 54-62.

Bogdanska, J., Sundström, M., Bergström, U., Borg, D., Abedi-Valugerdi, M., Bergman, А., DePierre, J., Nobel, S., 2014. Tissue distribution of 35S-labelled perfluorobutanesulfonic acid in adult mice following dietary exposure for 1-5 days. Chemosphere 98, 28-36.

Bogdanska, J., Borg, D., Bergström, U., Mellring, M., Bergman, Å., DePierre, J., Nobel, S., 2020. Tissue distribution of 14C-labelled perfluorooctanoic acid in adult mice after 1-5 days of dietary exposure to an experimental dose or a lower dose that resulted in blood levels similar to those detected in exposed humans. Chemosphere 239, 124755.

Borg, M., Brincat, S., Camilleri, G., Schembri-Wismayer, P., Brincat, M., Calleja-Agius, J., 2013. The role of cytokines in skin aging. Climacteric 16 (5), 514-521.

Brühl, C.A., Schmidt, T., Pieper, S., Alscher, A., 2013. Terrestrial pesticide exposure of amphibians: an underestimated cause of global decline? Sci. Rep. 3, 1135.

Buckingham, E.M., Klingelhutz, A.J., 2011. The role of telomeres in the ageing of human skin. Exp. Dermatol. 20 (4), 297-302.

Całyniuk, B., Grochowska-Niedworok, E., Walkiewicz, K.W., Kawecka, S., Popiołek, E. Fatyga, E., 2016. Malondialdehyde (MDA)-product of lipid peroxidation as marker of homeostasis disorders and aging. Ann. Acad. Med. Silesiensis 70, 224-228.

Cartwright, T., Perkins, N.D., Wilson, C.L., 2016. NFKB1: a suppressor of inflammation, ageing and cancer. FEBS J. 283 (10), 1812-1822.

Chang, A.L.S., Fu, T., Amir, O., Tang, J.Y., 2010. Association of facial skin aging and vitamin D levels in middle-aged white women. Cancer Causes \& Control 21 (12), 2315-2316.

Chang, S.-C., Ehresman, D.J., Bjork, J.A., Wallace, K.B., Parker, G.A., Stump, D.G., Butenhoff, J.L., 2009. Gestational and lactational exposure to potassium perfluorooctanesulfonate $(\mathrm{K}+\mathrm{PFOS})$ in rats: toxicokinetics, thyroid hormone status, and related gene expression. Reprod. Toxicol. 27 (3-4), 387-399.

Chen, Q., Huang, R., Hua, L., Guo, Y., Huang, L., Zhao, Y., Wang, X., Zhang, J., 2018 Prenatal exposure to perfluoroalkyl and polyfluoroalkyl substances and childhood atopic dermatitis: a prospective birth cohort study. Environ. Health 17 (1), 8.

Christensen, K.Y., Raymond, M., Blackowicz, M., Liu, Y., Thompson, B.A., Anderson, H. A., Turyk, M., 2017. Perfluoroalkyl substances and fish consumption. Environ. Res. $154,145-151$.

Chung, J.H., Seo, A.Y., Chung, S.W., Kim, M.K., Leeuwenburgh, C., Yu, B.P., Chung, H.Y., 2008. Molecular mechanism of PPAR in the regulation of age-related inflammation. Ageing Res. Rev. 7 (2), 126-136.

Coppé, J.-P., Desprez, P.-Y., Krtolica, A., Campisi, J., 2010. The senescence-associated secretory phenotype: the dark side of tumor suppression. Annu. Rev. Pathol. Mech. Dis. 5, 99-118.

Coquette, A., Berna, N., Vandenbosch, A., Rosdy, M., De Wever, B., Poumay, Y., 2003. Analysis of interleukin- $1 \alpha$ (IL- $1 \alpha$ ) and interleukin-8 (IL-8) expression and release in in vitro reconstructed human epidermis for the prediction of in vivo skin irritation and/or sensitization. Toxicol. Vitr. 17 (3), 311-321.

Cui, Q., Pan, Y., Zhang, H., Sheng, N., Wang, J., Guo, Y., Dai, J., 2018. Occurrence and tissue distribution of novel perfluoroether carboxylic and sulfonic acids and legacy per/polyfluoroalkyl substances in black-spotted frog (Pelophylax nigromaculatus). Environ. Sci. Technol. 52 (3), 982-990.

Danish, E., 2018. Risk assessment of fluorinated substances in cosmetic products. Survey of Chemical Substances in Consumer Products No. 169. EPA.

Debacq-Chainiaux, F., Erusalimsky, J.D., Campisi, J., Toussaint, O., 2009. Protocols to detect senescence-associated beta-galactosidase (SA- $\beta$ gal) activity, a biomarker of senescent cells in culture and in vivo. Nat. Protoc. 4 (12), 1798-1806.

Dérijard, B., Hibi, M., Wu, I.H., Barrett, T., Su, B., Deng, T., Karin, M., Davis, R.J., 1994 JNK1: a protein kinase stimulated by UV light and Ha-Ras that binds and phosphorylates the c-Jun activation domain. Cell 76 (6), 1025-1037.

DeWitt, J.C., 2015. Toxicological Effects of Perfluoroalkyl and Polyfluoroalkyl Substances. Springer.

Dong, G.-H., Liu, M.-M., Wang, D., Zheng, L., Liang, Z.-F., Jin, Y.-H., 2011. Sub-chronic effect of perfluorooctanesulfonate (PFOS) on the balance of type 1 and type 2 cytokine in adult C57BL6 mice. Arch. Toxicol. 85 (10), 1235-1244.

Dubrac, S., Schmuth, M., 2011. PPAR-alpha in cutaneous inflammation. Derm. Endocrinol. 3 (1), 23-26.

Elsner, P., Wiegand, C., Raschke, C., 2009. Skin aging: a brief summary of characteristic changes. Textbook of Aging Skin. Springer.

Esteva, F.J., Sahin, A.A., Smith, T.L., Yang, Y., Pusztai, L., Nahta, R., Buchholz, T.A., Buzdar, A.U., Hortobagyi, G.N., Bacus, S.S., 2004. Prognostic significance of phosphorylated P38 mitogen-activated protein kinase and HER-2 expression in lymph node-positive breast carcinoma. Cancer 100 (3), 499-506.

Etzel, T.M., Braun, J.M., Buckley, J.P., 2019. Associations of serum perfluoroalkyl substance and vitamin D biomarker concentrations in NHANES, 2003-2010. Int. J. Hyg. Environ. Health 222 (2), 262-269.

Fairley, K.J., Purdy, R., Kearns, S., Anderson, S.E., Meade, B., 2007. Exposure to the immunosuppresant, perfluorooctanoic acid, enhances the murine IgE and airway hyperreactivity response to ovalbumin. Toxicol. Sci. 97 (2), 375-383. 
Falk, S., Failing, K., Georgii, S., Brunn, H., Stahl, T., 2015. Tissue specific uptake and elimination of perfluoroalkyl acids (PFAAs) in adult rainbow trout (Oncorhynchus mykiss) after dietary exposure. Chemosphere 129, 150-156.

Farage, M.A., Miller, K.W., Elsner, P., Maibach, H.I., 2007. Structural characteristics of the aging skin: a review. Cutan. Ocul. Toxicol. 26 (4), 343-357.

Farage, M.A., Miller, K.W., Maibach, H.I., 2009. Textbook of Aging Skin. Springer Science \& Business Media.

Farage, M.A., Miller, K.W., Elsner, P., Maibach, H.I., 2013. Characteristics of the aging skin. Adv. Wound care 2 (1), 5-10.

Farage, M.A., Miller, K.W., Maibach, H.I., 2014. Degenerative changes in aging skin. In: Farage, M.A., Miller, K.W., Maibach, H.I. (Eds.), Textbook of Aging Skin. Springer, Berlin, Heidelberg, pp. 1-18.

Farage, M.A., Miller, K.W., Maibach, H.I., 2017. Degenerative changes in aging skin. In: Farage, M.A., Miller, K.W., Maibach, H.I. (Eds.), Textbook of Aging Skin. Springer, Berlin, Heidelberg, pp. 15-30.

Fasano, W.J., Kennedy, G.L., Szostek, B., Farrar, D.G., Ward, R.J., Haroun, L. Hinderliter, P.M., 2005. Penetration of ammonium perfluorooctanoate through rat and human skin in vitro. Drug Chem. Toxicol. 28 (1), 79-90.

Fouquerel, E., Barnes, R.P., Uttam, S., Watkins, S.C., Bruchez, M.P., Opresko, P.L., 2019. Targeted and persistent 8-oxoguanine base damage at telomeres promotes telomere loss and crisis. Mol. Cell 75 (1), 117-130 e6.

Franko, J., Meade, B., Frasch, H.F., Barbero, A.M., Anderson, S.E., 2012. Dermal penetration potential of perfluorooctanoic acid (PFOA) in human and mouse skin. J. Toxicol. Environ. Health, Part A 75 (1), 50-62.

Fumagalli, M., Rossiello, F., Clerici, M., Barozzi, S., Cittaro, D., Kaplunov, J.M., Bucci, G. Dobreva, M., Matti, V., Beausejour, C.M., Herbig, U., Longhese, M.P., d'Adda di Fagagna, F., 2012. Telomeric DNA damage is irreparable and causes persistent DNA damage-response activation. Nat. Cell Biol. 14 (4), 355-365.

Gannon, S.A., Johnson, T., Nabb, D.L., Serex, T.L., Buck, R.C., Loveless, S.E., 2011 Absorption, distribution, metabolism, and excretion of [1-14 C]-perfluorohexanoate ([14C]-PFHx) in rats and mice. Toxicology 283 (1), 55-62.

Ge, J., Wang, C., Nie, X., Yang, J., Lu, H., Song, X., Su, K., Li, T., Han, J., Zhang, Y., Mao, J., Gu, Y., Zhao, J., Jiang, S., Wu, Q., 2016. ROS-mediated apoptosis of HAPI microglia through p53 signaling following PFOS exposure. Environ. Toxicol. Pharmacol. 46, 9-16.

Genovese, L., Sibilla, S., 2015. Innovative nutraceutical approaches to counteract the signs of aging. Textbook of Aging Skin. Springer, Berlin.

Goeritz, I., Falk, S., Stahl, T., Schäfers, C., Schlechtriem, C., 2013. Biomagnification and tissue distribution of perfluoroalkyl substances (PFASs) in market-size rainbow trout (Oncorhynchus mykiss). Environ. Toxicol. Chem. 32 (9), 2078-2088.

Gordon, S.C., 2011. Toxicological evaluation of ammonium 4, 8-dioxa-3H-perfluorononanoate, a new emulsifier to replace ammonium perfluorooctanoate in fluoropolymer manufacturing. Regul. Toxicol. Pharmacol. 59 (1), 64-80.

Gu, Y., Han, J., Jiang, C., Zhang, Y., 2020. Biomarkers, oxidative stress and autophagy in skin aging. Ageing Res. Rev. 59, 101036.

Hagenaars, A., Vergauwen, L., Benoot, D., Laukens, K., Knapen, D., 2013. Mechanistic toxicity study of perfluorooctanoic acid in zebrafish suggests mitochondrial dysfunction to play a key role in PFOA toxicity. Chemosphere 91 (6), 844-856.

Han, J.-S., Jang, S., Son, H.-Y., Kim, Y.-B., Kim, Y., Noh, J.-H., Kim, M.J., Lee, B.S., 2020 Subacute dermal toxicity of perfluoroalkyl carboxylic acids: comparison with different carbon-chain lengths in human skin equivalents and systemic effects of perfluoroheptanoic acid in Sprague Dawley rats. Arch. Toxicol. 94 (2), 523-539.

Harris, S.L., Levine, A.J., 2005. The p53 pathway: positive and negative feedback loops. Oncogene 24 (17), 2899-2908.

Hawkins, S., Shingleton, W., Adamus, J., Meldrum, H., 2010. Peroxisome proliferatoractivated receptors: role in skin health and appearance of photoaged skin. In: Farage, Miller, Maibach (Eds.), Textbook of Aging Skin. Springer-Veriag, Berlin, Heidelberg.

Herbig, U., Ferreira, M., Condel, L., Carey, D., Sedivy, J.M., 2006. Cellular senescence in aging primates. Science 311 (5765), 1257.

Honda, M., Muta, A., Shimazaki, A., Akasaka, T., Yoshikuni, M., Shimasaki, Y., Oshima, Y., 2018. High concentrations of perfluorooctane sulfonate in mucus of tige puffer fish Takifugu rubripes: a laboratory exposure study. Environ. Sci. Pollut. Res. 25 (2), 1551-1558.

Hughes, M.F., 2017. Percutaneous penetration of chemicals and aging skin. In Farage, M.A., Miller, K.W., Maibach, H.I. (Eds.), Textbook of Aging Skin. Springer, Berlin, Heidelberg, pp. 1489-1502.

Hundley, S., Sarrif, A., Kennedy Jr, G., 2006. Absorption, distribution, and excretion of ammonium perfluorooctanoate (APFO) after oral administration to various species. Drug Chem. Toxicol. 29 (2), 137-145.

Kennedy Jr, G.L., 1985. Dermal toxicity of ammonium perfluorooctanoate. Toxicol. Appl Pharmacol. 81 (2), 348-355.

Koul, H.K., Pal, M., Koul, S., 2013. Role of p38 MAP kinase signal transduction in solid tumors. Genes Cancer 4 (9-10), 342-359.

Krutmann, J., Bouloc, A., Sore, G., Bernard, B.A., Passeron, T., 2017. The skin aging exposome. J. Dermatol. Sci. 85 (3), 152-161.

Li, R., Munoz, G., Liu, Y., Sauvé, S., Ghoshal, S., Liu, J., 2019. Transformation of novel polyfluoroalkyl substances (PFASs) as co-contaminants during biopile remediation of petroleum hydrocarbons. J. Hazard. Mater. 362, 140-147.

Li, Y., Men, B., He, Y., Xu, H., Liu, M., Wang, D., 2017. Effect of single-wall carbon nanotubes on bioconcentration and toxicity of perfluorooctane sulfonate in zebrafish (Danio rerio). Sci. Total Environ. 607, 509-518.

Liu, C., Yu, K., Shi, X., Wang, J., Lam, P., Wu, R., ZHOU, B., 2007. Induction of oxidative stress and apoptosis by PFOS and PFOA in primary cultured hepatocytes of freshwater tilapia (Oreochromis niloticus). Aquat. Toxicol. 82 (2), 135-143.
Liu, H., Chen, Q., Lei, L., Zhou, W., Huang, L., Zhang, J., Chen, D., 2018. Prenatal exposure to perfluoroalkyl and polyfluoroalkyl substances affects leukocyte telomere length in female newborns. Environ. Pollut. 235, 446-452.

Lu, Y., Luo, B., Li, J., Dai, J., 2016. Perfluorooctanoic acid disrupts the blood-testis barrier and activates the TNF $\alpha / \mathrm{p} 38$ MAPK signaling pathway in vivo and in vitro. Arch. Toxicol. 90 (4), 971-983.

Lunardi, D., Abelli, L., Panti, C., Marsili, L., Fossi, M.C., Mancia, A., 2016. Transcriptomic analysis of bottlenose dolphin (Tursiops truncatus) skin biopsies to assess the effects of emerging contaminants. Mar. Environ. Res. 114, 74-79.

Maggio, M., Guralnik, J.M., Longo, D.L., Ferrucci, L., 2006. Interleukin-6 in aging and chronic disease: a magnificent pathway. J. Gerontol. Ser. A: Biol. Sci. Med. Sci. 61 (6), 575-584.

Mah, L., El-Osta, A., Karagiannis, T., 2010. $\gamma$ H2AX: a sensitive molecular marker of DNA damage and repair. Leukemia 24 (4), 679-686.

Meng, D., Guo, M., Qian, Y., Han, G., 2017. Occurrence and dietary exposure assessment of PFOS and PFOA in cultured Trachinotus ovatus in China. J. Environ. Sci. Health Part B 52 (9), 690-698.

Mollenhauer, M.A., Carter, B.J., Peden-Adams, M.M., Bossart, G.D., Fair, P.A., 2009. Gene expression changes in bottlenose dolphin, Tursiops truncatus, skin cells following exposure to methylmercury ( $\mathrm{MeHg}$ ) or perfluorooctane sulfonate (PFOS). Aquat. Toxicol. 91 (1), 10-18.

Møller, P., Wils, R.S., Jensen, D.M., Andersen, M.H.G., Roursgaard, M., 2018. Telomere dynamics and cellular senescence: an emerging field in environmental and occupational toxicology. Crit. Rev. Toxicol. 48 (9), 761-788.

Morre, D.M., Morre, D.J., 2014. arNOX: a new source of aging. In: Farage, M.A., Miller, K.W., Maibach, H.I. (Eds.), Textbook of Aging Skin. Springer, Berlin, Heidelberg, pp. 1-10.

Morré, D.J., Morré, D.M., 2014. arNOX: new mechanisms of skin aging and lipoprotein oxidation. In: Farage, M.A., Miller, K.W., Maibach, H.I. (Eds.), Textbook of Aging Skin. Springer, Berlin, Heidelberg, pp. 1-13.

Mousavi, S.E., Amini, H., Heydarpour, P., Chermahini, F.A., Godderis, L., 2019. Air pollution, environmental chemicals, and smoking may trigger vitamin D deficiency: evidence and potential mechanisms. Environ. Int. 122, 67-90.

Nakayama, S.F., Yoshikane, M., Onoda, Y., Nishihama, Y., Iwai-Shimada, M., Takagi, M., Kobayashi, Y., Isobe, T., 2019. Worldwide trends in tracing poly-and perfluoroalkyl substances (PFAS) in the environment. TrAC Trends Anal. Chem. 121, 115410.

Okazaki, M., 2009. 38: Aging and melanocytes stimulating cytokine expressed by keratinocyte and fibroblast. Textbook of Skin Aging. Springer, p. 6.

Olsen, G.W., Mair, D.C., Church, T.R., Ellefson, M.E., Reagen, W.K., Boyd, T.M., Herron, R.M., Medhdizadehkashi, Z., Nobiletti, J.B., Rios, J.A., Butenhoff, J.L., Zobel, L.R., 2008. Decline in perfluorooctanesulfonate and other polyfluoroalkyl chemicals in American Red Cross adult blood donors, 2000-2006. Environ. Sci. Technol. 42 (13), 4989-4995.

Ouzzani, M., Hammady, H., Fedorowicz, Z., Elmagarmid, A., 2016. Rayyan-a web and mobile app for systematic reviews. Syst. Rev. 5 (1), 210.

Parrish, A.R., 2017. The impact of aging on epithelial barriers. Tissue Barriers 5 (4) e1343172.

Pearl, M., Poon, V., Eyles, D., Lyall, K., Kharrazi, M., Croen, L., Windham, G., 2018 Relation of maternal serum concentrations of persistent organic pollutants to vitamin D in Southern California. ISEE Conference Abstracts. ISEE.

Peres, P., Terra, V., Guarnier, F., Cecchini, R., Cecchini, A., 2011. Photoaging an chronological aging profile: Understanding oxidation of the skin. J. Photochem. Photobiol. B Biol. 103 (2), 93-97.

Pérez, F., Llorca, M., Köck-Schulmeyer, M., Škrbić, B., Oliveira, L.S., da Boit Martinello, K., Al-Dhabi, N.A., Antić, I., Farré, M., Barceló, D., 2014. Assessment of perfluoroalkyl substances in food items at global scale. Environ. Res. 135, 181-189.

Peropadre, A., Freire, P.F., Hazen, M.J., 2018. A moderate exposure to perfluorooctanoic acid causes persistent DNA damage and senescence in human epidermal HaCaT keratinocytes. Food Chem. Toxicol. 121, 351-359.

Piérard-Franchimont, C., Paquet, P., Quatresooz, P., Piérard, G., 2007. Smoothing the mosaic subclinical melanoderma by calcipotriol. J. Eur. Acad. Dermatol, Venereol. 21 (5), 657-661.

Pignotti, E., Casas, G., Llorca, M., Tellbüscher, A., Almeida, D., Dinelli, E., Farré, M., Barceló, D., 2017. Seasonal variations in the occurrence of perfluoroalkyl substances in water, sediment and fish samples from Ebro Delta (Catalonia, Spain). Sci. Total Environ. 607, 933-943.

Poothong, S., Padilla-Sánchez, J.A., Papadopoulou, E., Giovanoulis, G., Thomsen, C. Haug, L.S., 2019. Hand wipes: a useful tool for assessing human exposure to polyand perfluoroalkyl substances (PFASs) through hand-to-mouth and dermal contacts. Environ. Sci. Technol. 53 (4), 1985-1993.

Poothong, S., Papadopoulou, E., Padilla-Sánchez, J.A., Thomsen, C., Haug, L.S., 2020. Multiple pathways of human exposure to poly-and perfluoroalkyl substances (PFASs): from external exposure to human blood. Environ. Int. 134, 105244.

Popoola, O.K., Marnewick, J.L., Rautenbach, F., Ameer, F., Iwuoha, E.I., Hussein, A.A. 2015. Inhibition of oxidative stress and skin aging-related enzymes by prenylated chalcones and other flavonoids from Helichrysum teretifolium. Molecules 20 (4), 7143-7155.

Pusceddu, I., Farrell, C.-J.L., Di Pierro, A.M., Jani, E., Herrmann, W., Herrmann, M., 2015. The role of telomeres and vitamin D in cellular aging and age-related diseases. Clin. Chem. Lab. Med. (CCLM) 53 (11), 1661-1678.

Qazi, M.R., Bogdanska, J., Butenhoff, J.L., Nelson, B.D., DePierre, J.W., AbediValugerdi, M., 2009. High-dose, short-term exposure of mice to perfluorooctanesulfonate (PFOS) or perfluorooctanoate (PFOA) affects the number of circulating neutrophils differently, but enhances the inflammatory responses of macrophages to lipopolysaccharide (LPS) in a similar fashion. Toxicology 262 (3), 207-214. 
Qiu, L., Qian, Y., Liu, Z., Wang, C., Qu, J., Wang, X., Wang, S., 2016. Perfluorooctane sulfonate (PFOS) disrupts blood-testis barrier by down-regulating junction proteins via p38 MAPK/ATF2/MMP9 signaling pathway. Toxicology 373, 1-12.

Raj, U.L., Sharma, G., Dang, S., Gupta, S., Gabrani, R., 2017. Impact of dietary supplements on skin aging. In: Farage, M.A., Miller, K.W., Maibach, H.I. (Eds.), Textbook of Aging Skin. Springer, Berlin, Heidelberg, pp. 579-591.

Rattan, S.I.S., 2017. Aging and senescence of skin cells in culture. In: Farage, M.A., Miller, K.W., Maibach, H.I. (Eds.), Textbook of Aging Skin. Springer, Berlin, Heidelberg, pp. 1239-1246.

Redon, C., Pilch, D., Rogakou, E., Sedelnikova, O., Newrock, K., Bonner, W., 2002 Histone H2a variants H2AX and H2AZ. Curr. Opinion Genet. Dev. 12 (2), 162-169.

Reichenberger, E., Baur, S., Sukotjo, C., Olsen, B., Karimbux, N., Nishimura, I., 2000. Collagen XII mutation disrupts matrix structure of periodontal ligament and skin. J. Dent. Res. 79 (12), 1962-1968.

Reichrath, J., 2012. Unravelling of hidden secrets: the role of vitamin D in skin aging. Derm. -Endocrinol. 4 (3), 241-244.

Rinnerthaler, M., Duschl, J., Steinbacher, P., Salzmann, M., Bischof, J., Schuller, M., Wimmer, H., Peer, T., Bauer, J.W., Richter, K., 2013. Age-related changes in the composition of the cornified envelope in human skin. Exp. Dermatol. 22 (5), 329-335.

Saejia, P., Lirdprapamongkol, K., Svasti, J., Paricharttanakul, N.M., 2019. Perfluorooctanoic acid enhances invasion of follicular thyroid carcinoma cells through NF-кB and matrix metalloproteinase-2 activation. Anticancer Res. 39 (5), $2429-2435$.

Sandilands, A., Sutherland, C., Irvine, A.D., McLean, W.I., 2009. Filaggrin in the frontline: role in skin barrier function and disease. J. Cell Sci. 122 (9), 1285-1294.

Senthilkumar, P., Klingelhutz, A., Jacobus, J., Lehmler, H., Robertson, L., Ludewig, G., 2011. Airborne polychlorinated biphenyls (PCBs) reduce telomerase activity and shorten telomere length in immortal human skin keratinocytes (HaCat). Toxicol. Lett. 204 (1), 64-70.

Senthilkumar, P., Robertson, L., Ludewig, G., 2012. PCB153 reduces telomerase activity and telomere length in immortalized human skin keratinocytes (HaCaT) but not in human foreskin keratinocytes (NFK). Toxicol. Appl. Pharmacol. 259 (1), 115-123.

Shane, H.L., Baur, R., Lukomska, E., Weatherly, L., Anderson, S.E., 2020. Immunotoxicity and allergenic potential induced by topical application of perfluorooctanoic acid (PFOA) in a murine model. Food Chem. Toxicol. 136, 111114

Shay, J.W., Wright, W.E., 2019. Telomeres and telomerase: three decades of progress. Nat. Rev. Genet. 20 (5), 299-309.

Streubel, M.K., Rinnerthaler, M., Bischof, J., Richter, K., 2017. Changes in the composition of the cornified envelope during skin aging: a calcium centric point of view. In: Farage, M.A., Miller, K.W., Maibach, H.I. (Eds.), Textbook of Aging Skin. Springer, Berlin, Heidelberg, pp. 265-284

Su, H., Lu, Y., Wang, P., Shi, Y., Li, Q., Zhou, Y., Johnson, A.C., 2016. Perfluoroalkyl acids (PFAAs) in indoor and outdoor dusts around a mega fluorochemical industria park in China: implications for human exposure. Environ. Int. 94, 667-673.

Temkin, A.M., Hocevar, B.A., Andrews, D.Q., Naidenko, O.V., Kamendulis, L.M., 2020 Application of the key characteristics of carcinogens to per and polyfluoroalkyl substances. Int. J. Environ. Res. Public Health 17 (5), 1668.

\section{TUVW, 2016. Handout on Health: Atopic Dermatitis (A type of eczema).}

van de Sandt, J., Roguet, R., Cohen, C., Esdaile, D., Ponec, M., Corsini, E., Barker, C., Fusenig, N., Liebsch, M., Benford, D., de Brugerolle de Fraissinette, A., Fartasch, M.,
1999. The use of human keratinocytes and human skin models for predicting skin irritation: the report and recommendations of ECVAM workshop 38. Altern. Lab. Anim. 27 (5), 723-743.

Victorelli, S., Passos, J.F., 2020. Telomeres: beacons of autocrine and paracrine DNA damage during skin aging. Cell Cycle 19 (5), 532-540.

Wang, Y., Zhang, X., Wang, M., Cao, Y., Wang, X., Liu, Y., Wang, J., Wang, J., Wu, L. Hei, T.K., Luan, Y., Xu, A., 2015. Mutagenic effects of perfluorooctanesulfonic acid in GPT delta transgenic system are mediated by hydrogen peroxide. Environ. Sci. Technol. 49 (10), 6294-6303.

Wang, Z., Cousins, I.T., Scheringer, M., Hungerbühler, K., 2013. Fluorinated alternatives to long-chain perfluoroalkyl carboxylic acids (PFCAs), perfluoroalkane sulfonic acids (PFSAs) and their potential precursors. Environ. Int. 60, 242-248.

Wang, Z., Cousins, I.T., Scheringer, M., Hungerbuehler, K., 2015. Hazard assessment of fluorinated alternatives to long-chain perfluoroalkyl acids (PFAAs) and their precursors: status quo, ongoing challenges and possible solutions. Environ. Int. 75, $172-179$.

Wang, Z., DeWitt, J.C., Higgins, C.P., Cousins, I.T., 2017. A Never-Ending Story of Perand Polyfluoroalkyl Substances (PFASs)? ACS Publications,.

Wen, H.-J., Wang, S.-L., Chen, P.-C., Guo, Y.L., 2019. Prenatal perfluorooctanoic acid exposure and glutathione s-transferase T1/M1 genotypes and their association with atopic dermatitis at 2 years of age. Plos One 14 (1), e0210708.

Wen, H.-J., Wang, S.-L., Chuang, Y.-C., Chen, P.-C., Guo, Y.L., 2019. Prenatal perfluorooctanoic acid exposure is associated with early onset atopic dermatitis in 5year-old children. Chemosphere 231, 25-31.

Wielsøe, M., Long, M., Ghisari, M., Bonefeld-Jørgensen, E.C., 2015. Perfluoroalkylated substances (PFAS) affect oxidative stress biomarkers in vitro. Chemosphere 129, $239-245$.

Yahia, D., Haruka, I., Kagashi, Y., Tsuda, S., 2016. 8-Hydroxy-2'-deoxyguanosine as a biomarker of oxidative DNA damage induced by perfluorinated compounds in TK 6 cells, Fnviron. Toxicol, 31 (2), 192-200.

Yang, B., Zou, W., Hu, Z., Liu, F., Zhou, L., Yang, S., Kuang, H., Wu, L., Wei, J., Wang, J., Zou, T., Zhang, D., 2014. Involvement of oxidative stress and inflammation in liver injury caused by perfluorooctanoic acid exposure in mice. BioMed. Res. Int. 2014.

Yu, D., Li, M., Tian, Y., Liu, J., Shang, J., 2015. Luteolin inhibits ROS-activated MAPK pathway in myocardial ischemia/reperfusion injury. Life Sci. 122, 15-25.

Yu, Y., Wang, C., Zhang, X., Zhu, J., Wang, L., Ji, M., Zhang, Z., Ji, X.M., Wang, S.L., 2020. Perfluorooctane sulfonate disrupts the blood brain barrier through the crosstalk between endothelial cells and astrocytes in mice. Environ. Pollut. 256, 113429.

Zhang, W., Wang, F., Xu, P., Miao, C., Zeng, X., Cui, X., Lu, C., Xie, H., Yin, H., Chen, F., Ma, J., Gao, S., Fu, Z., 2014. Perfluorooctanoic acid stimulates breast cancer cells invasion and up-regulates matrix metalloproteinase-2/-9 expression mediated by activating NF-kB. Toxicol. Lett. 229 (1), 118-125.

Zhang, X., Lin, S., Funk, W.E., Hou, L., 2013. Republished: environmental and occupational exposure to chemicals and telomere length in human studies. Postgrad. Med. J. 89 (1058), 722-728.

Zhao, S., Liu, T., Wang, B., Fu, J., Liang, T., Zhong, Z., Zhan, J., Liu, L., 2019. Accumulation, biodegradation and toxicological effects of N-ethyl perfluorooctane sulfonamidoethanol on the earthworms Eisenia fetida exposed to quartz sands. Ecotoxicol. Environ. Saf. 181, 138-145. 